\title{
ADINKRAS, DESSINS, ORIGAMI, AND SUPERSYMMETRY SPECTRAL TRIPLES
}

\author{
MATILDE MARCOLLI AND NICK ZOLMAN
}

\begin{abstract}
We investigate the spectral geometry and spectral action functionals associated to 1D Supersymmetry Algebras, using the classification of these superalgebras in terms of Adinkra graphs and the construction of associated dessin d'enfant and origami curves. The resulting spectral action functionals are computed in terms of the Selberg (super) trace formula.
\end{abstract}

\section{INTRODUCTION}

In this paper we construct spectral geometries (spectral triples) and spectral action functionals associated to $1 \mathrm{D}$ supersymmetry algebras. We use the classification of [15] of these superalgebras in terms of combinatorial data of Adinkra graphs, in combination with recent results of [13], which show that an Adinkra graph determines geometric data:

- a compact Riemann surface $X$,

- a branched covering $f: X \rightarrow \mathbb{P}^{1}(\mathbb{C})$ ramified at $\{0,1, \infty\}$ (that is, a Belyi map),

- a spin structure on $X$,

- a structure of Super Riemann Surface on $X$.

The first two property specify a dessin d'enfant, in the sense of Grothendieck, given by the graph $f^{-1}(\mathcal{I})$, for $\mathcal{I}=[0,1]$, embedded in $X$.

We associate a spectral geometry to a given $1 \mathrm{D}$ supersymmetry algebra, by considering the canonical spectral triple $\left(\mathcal{C}^{\infty}(X), L^{2}\left(X, \mathbb{S}_{\mathfrak{s}}, D_{\mathfrak{s}}\right)\right.$ on the Riemann surface $X$, with spin structure $\mathfrak{s}$ and spinor bundle $\mathbb{S}_{\mathfrak{s}}$ and Dirac operator $\not_{\mathfrak{s}}$, where $X$ and $\mathfrak{s}$ are determined by the Adinkra of the 1D supersymmetry algebra, as in [13].

We show that the associate spectral action functional of the spectral triple can be computed using the Selberg trace formula for a finite index subgroup of a Fuchsian triangle group of the form $\Delta_{N, N, 2}$. For a special choice of the test function, the spectral action functional can be computed in terms of the Selberg zeta function, which in turn can be computed with the method of [41], using the thermodynamic formalism and the Ruelle transfer operator of a symbolic dynamics coding the geodesic flow on $X$.

We also show that, given the transfer operator for the Fuchsian triangle group $\Delta_{N, N, 2}$, the one for a finite index subgroup $H \subset \Delta_{N, N, 2}$ can be obtained by extending the symbolic dynamics and the transfer operator from the boundary of the upper half plane to its product with the coset space $P=\Delta_{N, N, 2} / H$.

The results of [13] show that the Adinkra classifying the 1D supersymmetry algebra also determines on $X$ the structure of a Super Riemann Surface. We refine the construction of the spectral geometry and the spectral action functional by incorporating this supersymmetry, and replacing the Dirac operator with the supersymmetric Dirac Laplacian, which is an operator proportional to $D \bar{D}$, where $D=\partial_{\theta}+\theta \partial_{z}$ on the Super Riemann Surface. 
The associated action functional is a super spectral action computed as a supertrace of this operator, regularized by a test function. We show that this action functional can be computed using the Selberg supertrace formula of [1].

We also show, by using the construction in 38 of origami curves associated to dessins d'enfant, that the Adinkra graph $A$ of a 1D supersymmetry algebra determines uniquely an origami curve $Y$, in which the graph $A$ embeds, with a choice of $2^{n}$ embeddings, where $n=\# E(A)$ is the number of edges. We then use a result of [36] showing that, for all Riemann surfaces $Y$ that admits a branched cover $p: Y \rightarrow E$, with $E$ an elliptic curve (hence in particular for all origami curves), it is possible to construct a family of metrics on $Y$, determined by compatibility conditions on the period matrix coming from the existence of the branched cover map to $E$. For each metric in this family, it is shown in [36] how to obtain an infinite family of eigenvalues and eigenvectors of the corresponding Laplacian. The resulting spectrum and spectral action functional behave more like the spectral action of tori and its computation can be approached in terms of a Poisson summation formula.

1.1. Supersymmetry and Spectral geometry. Noncommutative geometry has developed a broad approach to the construction of particle physics models and gravity models (see the overviews given in [46] and in the upcoming [34]). These model provide extensions of the minimal standard model of elementary particle physics, as well as modified gravity models in cosmology, obtained from the spectral action functional of a suitable (noncommutative) geometry. Among the extensions of the minimal standard model that have been considered so far with this method, the recent work [2] showed to what extent the Minimally Supersymmetric Standard Model (MSSM) can be incorporated and recovered from this general approach. The results of [2] provided a novel and important insight on noncommutative geometry and supersymmetry.

In the present paper, we consider a different type of supersymmetric models, much simpler in nature than the MSSM of particle physics, namely the 1-dimensional supersymmetry algebras, or $(1 \mid N)$-Superalgebras.

We use the classification of [15] in terms of Adinkra graphs, and the results of [13] associating to an Adinkra graph a dessin d'enfant and a Belyi map, in order to associate to a supersymmetry algebra a geometric object, a Riemann surface, or a Super Riemann Surface with a spin structure. We then use this geometric object to obtain a spectral triple associated to the supersymmetry algebra. It is given by the standard spectral triple of this spin geometry. We compute the associated spectral action functional. We also consider another construction, which is based on the relation between dessins d'enfant and origami curves as in [38, to construct another related geometry and compare their spectral properties.

While the models we consider here are not directly related to the particle physics models considered in [2], they provide a rich class of examples of physical models with supersymmetry that have an associated spectral geometry and spectral action functional. It would be interesting to further investigate how this point of view based on spectral triples and the spectral action relates, for example, to the real homotopy theory approach of [27], though this is beyond the scope of the present paper.

1.2. Supersymmetry Algebras. In the theory of Supersymmetry, the possible symmetries of a 4-dimensional quantum field theory (viewed as symmetries of the $S$-matrix) are 
classified by the Haag-Lopuszanski-Sohnius theorem, [22. This result shows that the possible symmetries consist of internal symmetries and a nontrivial extension of the Poincaré algebra: the supersymmetry algebras.

The off-shell supersymmetry algebras we will be considering here correspond to the case of a 1-dimensional space-time, with time coordinate $t$, and zero-dimensional space (supersymmetric quantum mechanics). We consider the $(1 \mid N)$ Superalgebras, generated by operators $Q_{1}, Q_{2}, \ldots, Q_{N}$ and $H$, where the $Q_{k}, k=1, \ldots, N$ are the supersymmetry generators, and $H=i \partial_{t}$. These operators are required to satisfy the following commutation relations:

$$
\begin{gathered}
{\left[Q_{k}, H\right]=0} \\
\left\{Q_{k}, Q_{\ell}\right\}=2 \delta_{k \ell} H .
\end{gathered}
$$

We consider representations of these operators, acting on bosonic and fermionic fields. Let $\left\{\phi_{1}, \ldots, \phi_{m}\right\}$ be a set of bosonic fields, given by commuting real valued functions, and $\left\{\psi_{1}, \ldots, \psi_{m}\right\}$ be a set of fermionic fields, given by anticommuting real (Grassman variable) valued functions. The off-shell condition means that the only relations are given by (1.1) and $(1.2)$ and no other equation holds imposing additional relations between these fields. We consider operators acting as

$$
\begin{aligned}
Q_{k} \phi_{a} & =c \partial_{t}^{\lambda} \psi_{b} \\
Q_{k} \psi_{b} & =\frac{i}{c} \partial_{t}^{1-\lambda} \phi_{a},
\end{aligned}
$$

for parameters $c \in\{-1,1\}$ and $\lambda \in\{0,1\}$. These representations clearly satisfy the relations (1.1) and 1.2 .

1.3. Adinkras. Recently, a graphical method for classifying the supersymmetry algebras was introduced by Faux and Gates, [15]. The resulting decorated bipartite graphs are called Adinkras. The mathematical and physical properties of Adinkras were extensively studied in recent years, see for instance [12] and [13. A good introduction to Adinkras for mathematicians is given in [47]. We recall here briefly a few basic facts about Adinkras, following the aforementioned references.

Let $A$ be a finite graph that is simple, namely it has no looping edges and no parallel edges. We denote by $V(A)$ and $E(A)$ the sets of vertices and edges of $A$.

An $N$-dimensional chromotopology is a finite connected simple graph, $A$ such that:

- $A$ is $N$-regular (all vertices have valence $N$ ) and bipartite.

- The elements of $E(A)$ are colored by $N$ colors, represented by elements of the set $\{1,2, \ldots, N\}$.

- Every vertex is incident to exactly one edge of each color.

- For any distinct colors $i$ and $j$, the edges in $E(A)$ with colors $i$ and $j$ form a disjoint union of 4-cycles.

In superalgebras, the two sets of vertices that form the bipartition correspond to "bosons" and "fermions". We color them white and black, respectively.

A ranking on a graph $A$ is a partial ordering on the set $V(A)$, determined by a function, $h: V(A) \rightarrow \mathbb{Z}$. One can represent the ranking on a graph as vertical placement of the vertices, that is, as a height function. 
A dashing on a graph $A$ is a function $d: E(A) \rightarrow \mathbb{Z} / 2 \mathbb{Z}$ that assigns to each edge a value 0 (solid) or 1 (dashed). A 4-cycle in a graph has an odd-dashing if it has an odd number of dashed edges. A colored graph whose 2-colored 4-cycles all have an odd-dashing is called well-dashed.

An Adinkra is a well-dashed, $N$-dimensional chromotopology, with a ranking on its bipartition, such that the bosons have even ranking and the fermions have odd ranking. We call any chromotopology Adinkraizable if it can be well-dashed and it admits a well-defined ranking as above.

The results of [15] showed that Adinkras can be used to classify the one-dimensional superalgebras and to generate large classes of significant examples of such algebras. We just recall here briefly how one encodes a superalgebra of the form (1.3) and (1.4) as an Adinkra graph.

Given $\left\{\phi_{1}, \ldots, \phi_{m}\right\}$ (bosonic fields) and $\left\{\psi_{1}, \ldots, \psi_{m}\right\}$ (fermionic fields) with a representation of the form (1.3) and (1.4), with $c \in\{-1,1\}$ and $\lambda \in\{0,1\}$, as above, one constructs an Adinkra graph with a set of white vertices corresponding to the set of bosonic fields and time derivatives and a set of black vertices corresponding to the fermionic fields and time derivatives. An edge is assigned between a pair $\left\{v_{a}, v_{b}\right\}$ of vertices for each relation of the form (1.3) or 1.4), with the rule that the edge is oriented from the white to the black vertex in the pair if $\lambda=0$ and from the black to the white vertex if $\lambda=1$. Moreover, the edge is dashed if $c=-1$ and solid if $c=1$. This orientation is obtained by effect of the ranking function $h: V(A) \rightarrow \mathbb{Z}$ described above. We can summarize these rules on ranking and dashing in the following table.

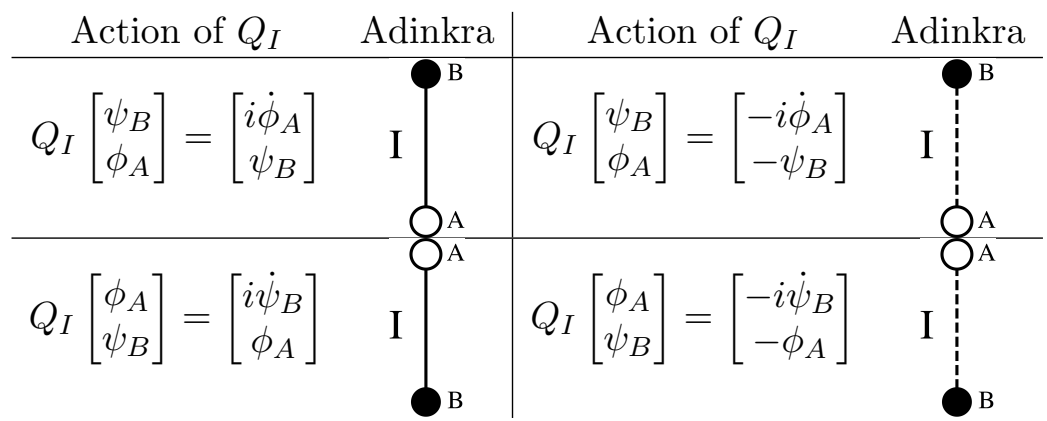

We refer the reader to [15], and [10], [11, [12], [13] for more details on the correspondence between Adinkras and one-dimensional superalgebras.

1.3.1. Adinkras from Codes. The simplest example of an Adinkra is provided by the $N$ cube $A_{N}$, whose vertices, identified with binary words of length $N$, one can think of as the Hamming cube. After labeling the $2^{N}$ vertices with the corresponding binary words, one connects with an edge a pair of vertices whose words differ by a single entry (words with Hamming distance 1). The resulting graph can be colored by assigning color $i$ to an edge connecting vertices whose words differ at the $i$-th binary digit. A ranking is obtained by defining $h: V(A) \rightarrow \mathbb{Z}$ as $h(v)=\#$ of 1 's in $v$. The bipartion into bosons and fermions is obtained by separating vertices with even ranking (bosons) and odd ranking (fermions). There are then $2^{2^{N}-1}$ possible choices of dashings. An example of a well-dashed 2-cube is given in Figure 1 .

It was shown in Theorem 4.4 of [10] (see also [11]) that all Adinkraizable chromotopologies can be obtained from the cube Adinkras via linear codes. Theorem 4.5 of [47] gives a slightly 


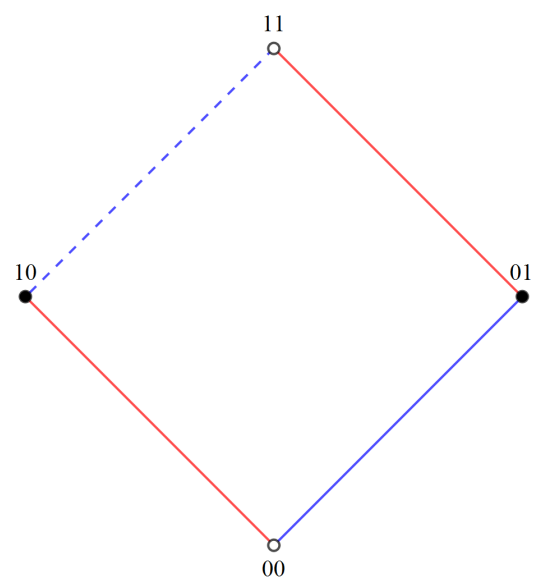

Figure 1. 2-cube Adinkra.

more general version of this result that includes multigraphs. More precisely, a linear binary code $L$ of dimension $k$ is a $k$-dimensional linear subspace of the $\mathbb{Z} / 2 \mathbb{Z}$-vector space $(\mathbb{Z} / 2 \mathbb{Z})^{N}$. The weight wt $(c)$ of codewords $c \in L$ counts the number of 1's in the codeword. A code $L$ is even if every $c \in L$ has even weight and doubly-even if the weight of every codeword is divisible by 4 . One can associate a chromotopology $A=A_{N} / L$ to the quotient space $(\mathbb{Z} / 2 \mathbb{Z})^{N} / L$ by taking as set of vertices $V(A)$ the equivalence class of vertices, with an edge of color $i$ connecting two classes $[v]$ and $[w] \in V(A)$ whenever there is at least one edge of color $i$ between a vertex $v^{\prime} \in[v]$ and a vertex $w^{\prime} \in[w]$. The properties of the code $L$ determine properties of the resulting chromotopology $A=A_{N} / L$. In particular, the following properties are significant to the construction of Adinkras:

- the graph $A$ has a loop iff L contains a codeword of weight 1.

- the graph $A$ has a double edge iff L contains a codeword of weight 2 .

- the graph $A$ can be ranked iff $A$ is bipartite, which is true iff $L$ is an even code.

- the graph $A$ can be well-dashed iff $L$ is a doubly-even code.

The main result of Theorem 4.4 of [10] then shows that Adinkraizable chromotopologies are exactly quotients $A=A_{N} / L$, with $L$ is a doubly-even linear code.

One often denotes by $A_{N, k}=A_{N} / L$ an Adinkra obtained from a code with $k=\operatorname{dim}(L)$.

\section{Adinkras, Dessins and Origami Curves}

In the recent paper 13, the authors uncovered a surprising connection between Adinkras and Grothendieck's theory of dessins d'enfants by showing that the data of an Adinkra diagram determine a Belyi pair.

According to Belyi's theorem [3], a smooth projective algebraic curve $X$ is defined over a number field if and only if it admits a Belyi map, that is, a branched cover $f: X \rightarrow \widehat{\mathbb{C}}=$ $\mathbb{P}^{1}(\mathbb{C})$ that is ramified only at the points 0,1 , and $\infty$. Moreover, let $X$ be a smooth algebraic curve defined over a number field and let $f: X \rightarrow \widehat{\mathbb{C}}$ be a Belyi function. Denote by $p, q, r$, positive common multiples of the orders of ramifications of $f$ at $0,1, \infty$, respectively, so that $p^{-1}+q^{-1}+r^{-1}<1$. Let $\Delta=\Delta_{p, q, r}$ be the Fuchsian triangle group of signature $(p, q, r)$. Then it is known (see [8]) that there exists a uniformization $\Phi: \mathbb{H} \rightarrow X=H \backslash \mathbb{H}$, where $\mathbb{H}$ 
is the hyperbolic upper half plane, ramified at $f^{-1}\{0,1, \infty\}$, and where the uniformizing group is a finite index subgroup $H \subset \Delta$. The Belyi function then gives a branched covering $f: X=H \backslash \mathbb{H} \rightarrow \widehat{\mathbb{C}}=\Delta_{p, q, r} \backslash \mathbb{H}$. If $p, q, r$ are the ramification numbers at every point of $f^{-1}(0), f^{-1}(1), f^{-1}(\infty)$, respectively, then $\Phi$ is the unramified universal cover of $X$.

2.1. Dessins d'enfant. Let $X$ be a compact Riemann surface and let $\widehat{\mathbb{C}}=\mathbb{P}^{1}(\mathbb{C})$ denote the Riemann sphere. A Belyi map $f: X \rightarrow \widehat{\mathbb{C}}$ is a meromorphic function, unramified outside of the set $\{0,1, \infty\}$. A dessin d'enfant (sometimes simply referred to as dessin) is a bipartite graph $\Gamma$ embedded on the surface $X$, obtained by placing white vertices at the points $f^{-1}(1)$, black vertices at the points $f^{-1}(0)$, and curvilinear edges along the preimage $f^{-1}\left(\mathcal{I}^{o}\right)$ of the open interval $\mathcal{I}^{o}=(0,1)$. A Belyi pair $(X, f)$ is a Riemann surface $X$ equipped with a Belyi map $f$. It should be noted that every Belyi pair defines a dessin and every dessin defines a Belyi pair.

Example 2.1. Consider the function $f(x)=-\frac{(x-1)^{3}(x-9)}{64 x}$. Figure 2 shows the associated dessin.

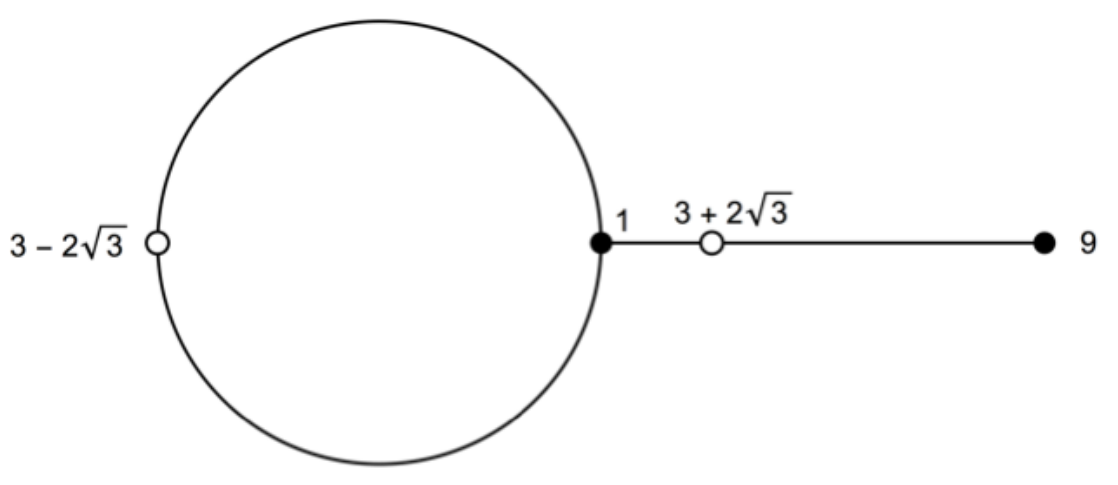

FIGURE 2. The dessin corresponding to the equation $f(x)=-\frac{(x-1)^{3}(x-9)}{64 x}$

It is proved in [13] that, given an Adinkra $A_{N, k}$, one can associate to it a dessin. This is achieved by constructing an embedding of $A_{N, k}$ on a compact Riemann surface $X_{N, k}$, of genus $g=1+2^{N-k-3}(N-4)$ for $N \geq 2$ and $g=0$ for $N<2$. The surface $X_{N, k}$ is constructed by attaching 2-cells to all consecutively colored 4-cycles of the Adinkra. Moreover, it is shown in [13] how to obtain the Belyi pair $f: X_{N, k} \rightarrow \hat{\mathbb{C}}$ with the Adinkra as dessin graph.

It was shown in [43] that a smooth projective algebraic curve is defined over a number fields if and only if, as a compact Riemann surface, it can be triangulated by equilateral triangles. More precisely, such a triangulation consists of an even number of open cells $T_{j}^{ \pm}$ homeomorphic to Euclidean triangles. The triangulation of $X$ lifts to a triangulation of the universal cover $\mathbb{H}$, such that, for each triangle $\tilde{T}$ in $\mathbb{H}$ there are reflections $\sigma_{1}, \sigma_{2}, \sigma_{3}$ in the sides of $\tilde{T}$ that are anti-holomorphic homeomorphisms of $\mathbb{H}$ preserving the triangulation, mapping the triangle $\tilde{T}$ to its neighbor sharing the side around which the reflection happens, and switching orientation. This is called a covered symmetric triangulation (see [8]). Given a Belyi map $f: X \rightarrow \widehat{\mathbb{C}}$, a covered symmetric triangulation is obtained by considering the 
two hemispheres $\mathbb{H}^{ \pm}$with $\mathbb{H}^{+} \cup \mathbb{H}^{-}=\mathbb{P}^{1}(\mathbb{C}) \backslash \mathbb{P}^{1}(\mathbb{R})$ and the triangles $T_{j}^{ \pm}$in $X$ given, respectively, by the connected components of $f^{-1}\left(\mathbb{H}^{ \pm}\right)$.

Lemma 2.2. For $N>4$, an Adinkra $A_{N, k}$ determines a covered symmetric triangulation on the Riemann surface $X_{N, k}$ with Fuchsian triangle group $\Delta=\Delta_{N, N, 2}$.

Proof. As shown in [13] the Belyi map $f: X_{N, k} \rightarrow \widehat{\mathbb{C}}$ has ramification of order $N$ at 0 and 1 and ramification of order 2 at $\infty$, hence according to [8] the Fuchsian triangle group associated to the dessin is $\Delta_{N, N, 2}$. The condition $N>4$ ensures the hyperbolic condition $\frac{2}{N}+\frac{1}{2}<1$ for the triangle Fuchsian groups. The remaining cases with $N \leq 4$ correspond to genus zero and genus one surfaces. The preimages $f^{-1}\left(\mathbb{H}^{ \pm}\right)$under the Belyi maps constitute the triangles $T_{j}^{ \pm}$of the covered symmetric triangulation. In $X_{N, k}$ the pairs of triangles $T_{j}^{+} \cup T_{j}^{-}$constitute the 2-cells attached to $A_{N, k}$ along consecutively colored 4-cycles in the Adinkra: the diagonal along which the two triangles are joined lies in the preimage under the Belyi map $f$ of the two arcs $(1, \infty)$ and $(\infty, 0)$ in $\mathbb{P}^{1}(\mathbb{R})=\mathbb{P}^{1}(\mathbb{C}) \backslash\left(\mathbb{H}^{+} \cup \mathbb{H}^{-}\right)$, with the preimage of the point $\infty$ lying in the middle of the 2-cell.

2.2. Origami curves. In addition to Grothendieck's theory of dessins d'enfant, another construction of algebraic curves based on combinatorial data has received considerable attention: the theory of origami curves. These consist of branched coverings $p: Y \rightarrow E$ ramified only over $\{\infty\}$. Origami curves determine Teichmüller embeddings of the hyperbolic plane in the Teichmüller space $T_{g, n}$ for given genus $g$ with $n$ marked points. These are complex geodesics (Teichmüller disks). Origami curves have been largely studied in connection to Teichmüller curves and Veech groups, see [24], 38]. Moreover, both dessins d'enfant and origami curves have been studied extensively as possible combinatorial objects carrying an action of the absolute Galois group, with the goal of gaining a better understanding of the absolute Galois group through combinatorial models of its action. In particular, various relations between dessins and origami have been studied, see [24], 38]. For our purposes, we will especially focus on a construction, given in 38] that associates an origami curve to a dessin d'enfant.

Let $E$ be an elliptic curve and let $X$ be a compact Riemann surface. An origami is a finite covering map $p: X \rightarrow E$ ramified over a single point $\infty \in E$.

Consider the cell structure of $E$ given by the generators $x, y$ shown in Figure 3 . Now consider the preimage $p^{-1}(E)$. This creates a finite cell structure on $X$ obtained by gluing cells together, as in Figure 4 .

Proposition 5.1 of [24] gives the following equivalent description of origami curves. An origami $p: X \rightarrow E$ is up to equivalence uniquely determined by

(1) A surface obtained from gluing Euclidean unit squares such that:

- each left edge is uniquely glued to a right edge and vice versa.

- each upper edge is glued to a unique lower one and vice versa.

- the result is a connected surface $X$.

(2) A finite oriented graph with edges labelled by $x$ and $y$, with the property that each vertex has exactly two incoming edges and two outgoing edges, with one of which type labelled, respectively, by $x$ and $y$.

(3) A monodromy map $\alpha: F_{2} \rightarrow S_{d}$, up to conjugation in $S_{d}$, where $F_{2}$ is the free group with 2 generators and $S_{d}$ is the permutation group of $d$ elements. 

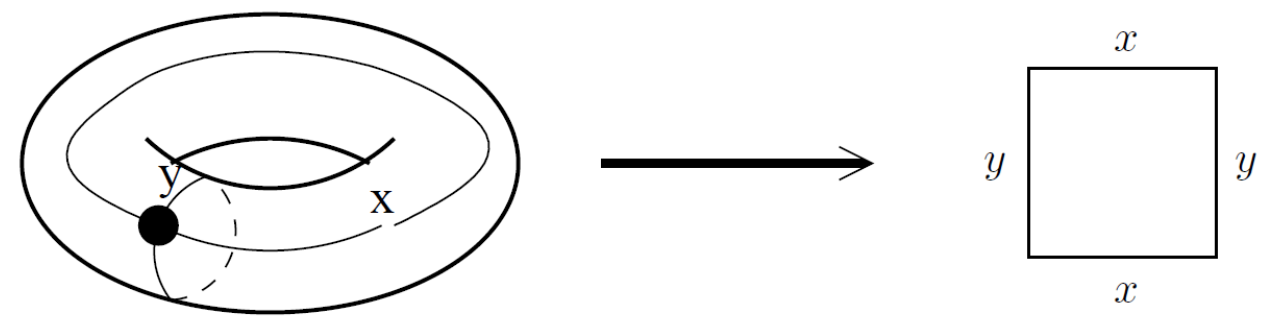

FiguRE 3. The torus with generators $x, y$. Reproduced from 24$]$

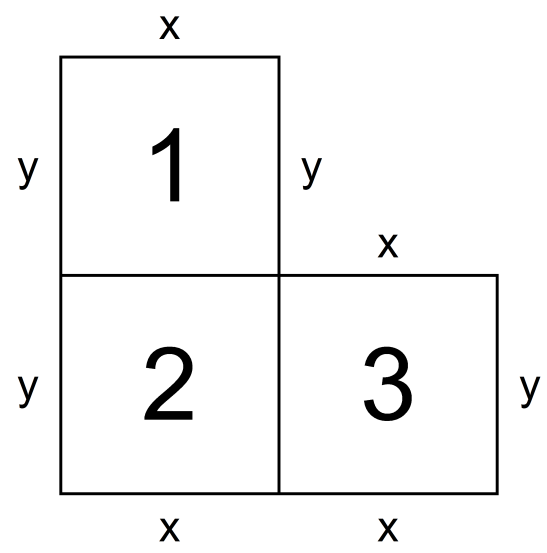

Figure 4. A simple example of an origami with opposite edges glued together.

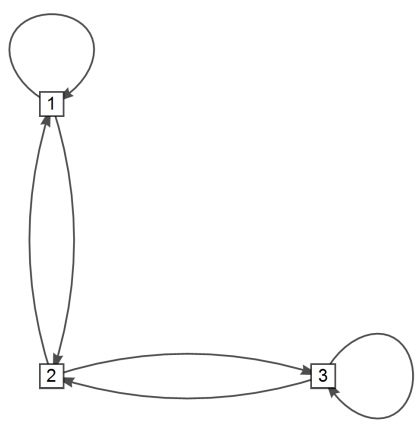

FiguRE 5. The oriented graph descrption of the origami in Figure 4

(4) A finite subgroup $U$ of $F_{2}$ up to conjugation in $F_{2}$.

2.3. Dessins and Origami curves. We recall here a way of passing from a dessin (a Belyi map $f: X \rightarrow \widehat{\mathbb{C}}$ ) to an origami curve, as introduced by [38] (see also $\S 4$ of [40]). This construction associates to a Belyi map $f: X \rightarrow \widehat{\mathbb{C}}$ an origami $p: Y \rightarrow E$, where the curve $Y$ is obtained by first taking the fibered product $\tilde{Y}=E \times_{\widehat{\mathbb{C}}} X$, fibered with respect to the map $f: X \rightarrow \widehat{\mathbb{C}}$ and the quotient $h: E \rightarrow \widehat{\mathbb{C}}$ by the elliptic involution,

$$
\tilde{Y}=E \times_{\widehat{\mathbb{C}}} X=\{(z, w) \in E \times X: h(z)=f(w)\} .
$$

The map $h: E \rightarrow \widehat{\mathbb{C}}$ is a double cover, ramified at four points $\{0,1, \infty, \lambda\}$. The curve $\tilde{Y}$ has singularities at those points $(z, w)$ such that $h$ is ramified at $z$ and $f$ is ramified at $w$. The desingularization $Y \rightarrow \tilde{Y}$ is connected and endowed with a map, which we still denote by $h$, to the elliptic curve, $h: Y \rightarrow E$, which is a branched covering, branched at $\{0,1, \infty, \lambda\}$. Let $m_{2}: E \rightarrow E$ be the multiplication by 2 , the unramified covering that maps four Weierstrass points (which we identify with $\{0,1, \infty, \lambda\}$ ) to $\infty$. Then the composition $p=m_{2} \circ h: Y \rightarrow E$ is ramified at only the point $\infty$ and is therefore an origami curve. 
One refers to the origami curve obtained in this way as the M-origami $O(f)=(p: Y \rightarrow E)$ associated to the dessin $f: X \rightarrow \widehat{\mathbb{C}}$.

2.4. 4-colored Adinkras and elliptic curves. By the general result of [13], all $A_{N, k}$ Adinkras give rise to dessins, which determine a Riemann surface $X_{N, k}$ and a Belyi map $f: X_{N, k} \rightarrow \widehat{\mathbb{C}}$, so that $A_{N, k}$ embeds in $X_{N, k}$ as the dessin obtained as the preimage $f^{-1}(\mathcal{I})$ with $\mathcal{I}=[0,1] \subset \widehat{\mathbb{C}}$.

Lemma 2.3. In the case of Adinkras $A_{N, k}$ with $N=4$ the Riemann surface $X_{4, k}$ is an elliptic curve.

Proof. For $N=4$, the genus formula $g=1+2^{N-k-3}(N-4)$ for $X_{N, k}$ immediately implies that $X_{4, k}$ is an elliptic curve.

We assign the colors bijectively to the directed edges. Because each vertex has four edges going to unique vertices, this translates to each face being adjacent to four unique faces in the origami picture, resulting in a grid cell structure. This corresponds exactly to the cell structure we would get if we glued 2-cells to consecutively colored 2-colored 4-cycles, as is done in [13] to determine the dessin. The choice of consecutively colored 2-cycles gives us an orientation, just as the directed edges do. By identifying edges according to labels we obtain a quotient $E$ that is still topologically a 2-torus, see Figures 6 and 7 , depicting the cases of the Adinkras $A_{4,0}$ and $A_{4,1}$, respectively.

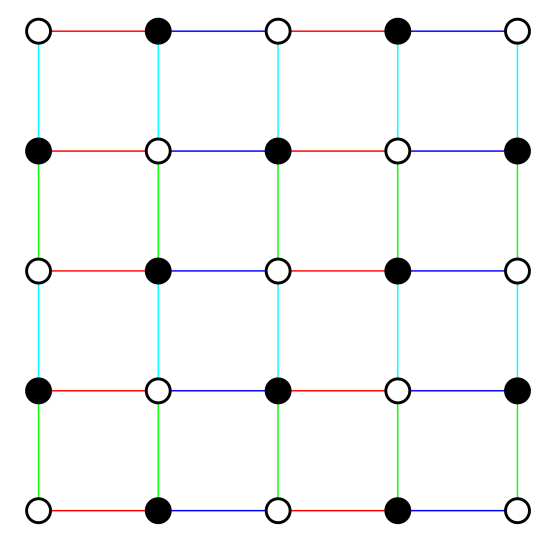

Figure 6 . The Adinkra $A_{4,0}$ embedded in an elliptic curve, obtained by identifying the top row of edges with the bottom row, and the left column of edges with the right column.

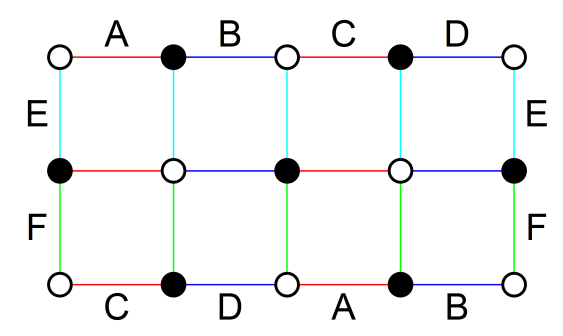

Figure 7 . The Adinkra $A_{4,1}$ embedded in an elliptic curve, obtained by identifying the edges as labeled.

2.5. Dual graphs of Adinkras and origami. We present here a relation between Adinkras and origami curves that uses the characterization recalled above (Proposition 5.1 of [24]) of origami in terms of oriented graphs with edges labeled $x$ or $y$, two incoming and two outgoing edges at each vertex, with one of each type labelled by $x$ and $y$, respectively. We refer to an oriented graph satisfying these property as an "origami graph". 
Proposition 2.4. For $N>2$, let $A=A_{N, k}$ be an Adinkra, embedded on the Riemann surface $X_{N, k}$, formed by attaching 2-cells to all consecutively colored 4-cycles in A. Let $A^{*}$ denote the dual graph of $A$, with respect to the given embedding $A \hookrightarrow X_{N, k}$. If $N$ is even, then there exists an edge labeling and an orientation such that $A^{*}$ is an origami graph.
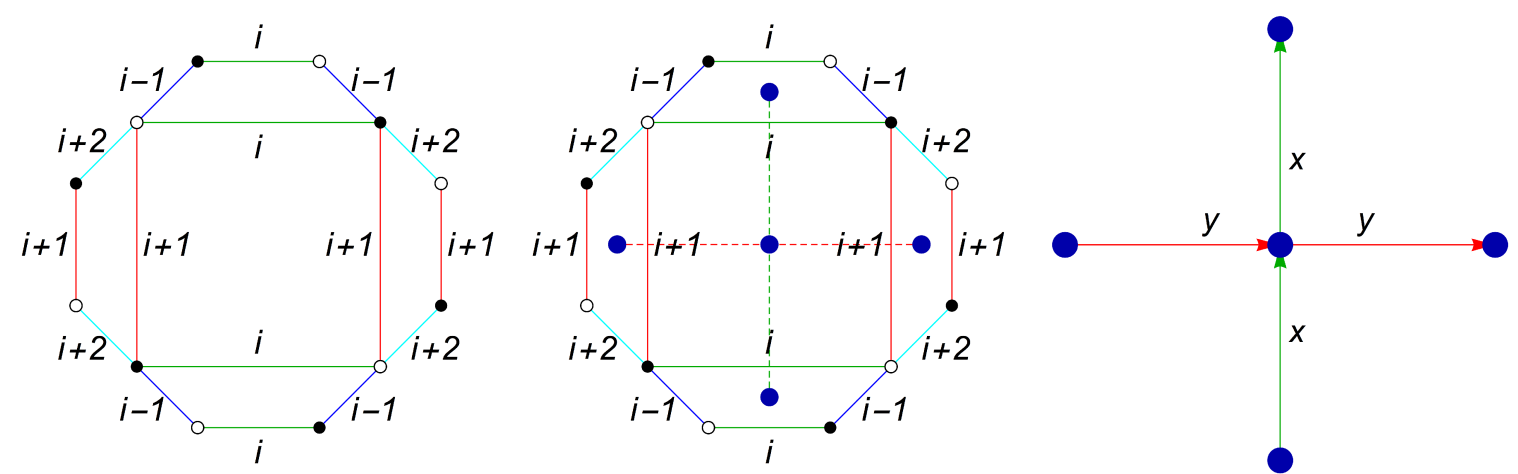

Figure 8. The process of defining the origami. Left: the Adinkra $A$ with face $F_{i, i+1}$ adjacent to faces with edges $i-1$ and $i+2$. Middle: The Adinkra, $A$, with its dual graph, $A^{*}$. Right: The oriented dual graph, $A^{*}$, with odd edges labeled $x$ and even edges labeled $y$. The resulting orientation and labeling is consistent with that of an origami.

Proof. Let $E$ and $V$ denote, respectively, the edge and vertex set of $A$. Similarly, let $E^{*}$ and $V^{*}$ denote the edge and vertex set of the dual graph $A^{*}$. First observe that, because every face of the embedding $A \hookrightarrow X_{N, k}$ is comprised of a consecutively colored 4-cycle, every vertex in $V^{*}$ has degree four. Let $F_{i, i+1}$ be a face in the embedding $A \hookrightarrow X_{N, k}$ with boundary a consecutively colored 4 -cycle with colors $\{i, i+1\}$. Then each edge with color $i$ in $\partial F_{i, i+1}$ is also incident to a face $F_{i-1, i}$ with colors $\{i-1, i\}$. Clearly these faces exist, as each vertex in $V$ is incident to exactly one edge of each color, and these faces are distinct, because if they were the same face, then there would exist a 2-cycle between the colors $\{i-1, i\}$ which is disallowed by the chromotopology rules. By the same argument, each edge of color $i+1$ in $\partial F_{i, i+1}$ is also incident to a face $F_{i+1, i+2}$ with boundary a 4 -cycles with colors $\{i+1, i+2\}$. Notice also that no other faces can be adjacent to $F_{i, i+1}$ because if there were other such faces $F_{i, j}$, then either there would be two edges of the same color incident to a vertex, or there would be a face formed by two non-consecutive colors. Neither of these possibilities is allowed by hypothesis. Thus, each face $F_{i, i+1}$ is incident to exactly four other faces. Thus, the dual graph $A^{*}$ is 4-regular. Now let us label the dual graph edges as follows. An edge $e^{*} \in E^{*}$ adjacent to a given vertex $v^{*} \in V^{*}$ crosses exactly one edge $e$ of the face $F_{i, i+1}$ of the embedding $A \hookrightarrow X_{N, k}$ that determines the vertex $v^{*}=v^{*}\left(F_{i, i+1}\right)$ of the dual graph. We label the edge $e^{*}$ with $x$ or $y$, according to whether the edge $e$ it crosses is colored by an odd color (i.e. $i=1,3,5 \ldots$ ), or an even color, respectively. Since the edges of the face of $A$ are consecutively colored, there are exactly two $x$-labelled edges and two $y$-labelled edges adjacent to each vertex $v^{*} \in V^{*}$. We need the assumption that $N$ is even: if $N$ were odd, then the edges adjacent to vertices in $V^{*}$ corresponding to faces $F_{1, N}$ with colors $\{1, N\}$ would all be labeled by $x$. To show that $A^{*}$ is an origami graph, we still need 
to assign an orientation to the graph, in such a way that at each vertex one incoming and one outgoing edge are labeled, respectively, by $x$ and $y$. Pick an arbitrary vertex and assign a valid orientation to each edge (with one incoming and outgoing edge labelled $x$ and $y$, respectively). Proceed inductively by following the outgoing edge of a given label: at the next vertex, by our construction, we know there is another edge labelled $y$, which we can orient outwards. The case of the $x$-labelled edges is analogous. This process terminates once a cycle has been created. Then proceed to the next undirected vertex. Note that there cannot exist two conflicting cycles, as this would imply that a vertex in $V^{*}$ has more than two edges of a given label, resulting in a contradiction. The construction of the origami associated to the dual graph is illustrated in Figure 8.

This construction generalizes the case of the $N=4$ Adinkras described in the previous Section 2.4, which corresponds to the self-dual graphs. In the rest of this section we describe a different construction that associates origami curves to Adinkras.

2.6. Dimers and spin curves. The starting point for the construction of spectral triples associated to $1 D$-supersymmetry algebras is the work of Cimasoni and Reshetikhin [7] on dimers on surface graphs and spin structures.

A perfect matching on a bipartite graph $A$ is a collection of edges such that every vertex is incident to exactly one edge of the collection. Perfect matchings are also referred to as dimer configurations. As observed in [13], if $A$ is an Adinkra, then taking the set of edges of a fixed color in $A$ determines a dimer configuration.

Let $A$ be a graph embedded on a compact Riemann surface $X$. A Kasteleyn orientation is an orientation of the edges of $A$ with the property that, when going around the boundary of a face counterclockwise, one goes against the orientation of an odd number of edges. As observed in [13], an odd dashing of an Adinkra $A_{N, k}$ determines a Kasteleyn orientation, when viewing the Adinkras as embedded in a Riemann surface, $A_{N, k} \hookrightarrow X_{N, k}$, as recalled above.

A vertex change operation on an Adinkra is a transformation that interchanges the dash/solid coloring of each edge incident to a chosen vertex. Two choices of dashing on an Adinkra are equivalent if they can be obtained from one another by performing a sequence of vertex changes.

It is shown in [7] (see also [13]) that a dimer configuration on an embedded graph $A$ on a Riemann surface $X$ determines an isomorphism between Kasteleyn orientations on $A$ (up to equivalence) and spin structures on $X$. Equivalent dashings give equivalent orientations, which correspond to the same spin structure on $X$.

2.7. Products and fibered products. In addition to the Adinkras considered above, one can include other combinatorial objects obtained from Adinkras via various types of operations on graphs. For instance, a Cartesian product (with additional structure that prescribes coloring, ranking, and dashing) was considered in [18], 25] and [26], for $2 \mathrm{D}$ supersymmetry algebras. These products of Adinkras are defined in the following way. Let $A_{1}=A_{N_{1}, k_{1}}$ and $A_{2}=A_{N_{2}, k_{2}}$ be two Adinkras with colors $1,2, \ldots, N_{1}$ and $N_{1}+1, N_{1}+$ $2, \ldots, N_{1}+N_{2}$, respectively. The product $A_{1} \times A_{2}$ is defined as follows. Let $u, v \in V\left(A_{1}\right)$ and $u^{\prime}, v^{\prime} \in V\left(A_{2}\right)$. We denote the presence of an edge between vertices $v_{1}, v_{2}$ with the notation $v_{1} \sim v_{2}$. We also denote the color of a vertex by $c_{i}(v) \in\{-1,1\}$, where 1 is white and -1 is black. For $\left(u, u^{\prime}\right),\left(v, v^{\prime}\right) \in V\left(A_{1} \times A_{2}\right)$ we have (see [26]): 
- $\left(u, u^{\prime}\right) \sim\left(v, v^{\prime}\right)$ with color $i$ if and only if:

$-u \sim v$ with color $i$ and $u^{\prime}=v^{\prime}$

- or $u=v$ and $u^{\prime} \sim v^{\prime}$ with color $i$.

- the vertex color of $\left(u, u^{\prime}\right)$ is given by $c\left(u, u^{\prime}\right)=c_{1}(u) c_{2}\left(u^{\prime}\right)$.

- the ranking of $\left(u, u^{\prime}\right)$ is given by $h\left(u, u^{\prime}\right)=h_{1}(u)+h_{2}\left(u^{\prime}\right)$, where $h_{i}$ are the rankings of each Adinkra.

- the dashing $d\left(\left(u, u^{\prime}\right) \sim\left(v, v^{\prime}\right)\right)$ of edges in the product Adinkra is given by $d_{1}(u \sim$ $u^{\prime}$ ), the dashing of $A_{1}$, or by $d_{2}\left(v \sim v^{\prime}\right)+h_{1}(u) \bmod 2$, with $d_{2}$ the dashing of $A_{2}$, in the two cases of $\left(u, u^{\prime}\right) \sim\left(v, v^{\prime}\right)$ with $u^{\prime}=v^{\prime}$ and $u=v$, respectively.

We consider here a different kind of product of Adinkras, which we view as a fibered product, because they will correspond to taking fibered products of the corresponding Riemann surfaces fibered over the Belyi maps.

Definition 2.5. Let $A_{1}$ and $A_{2}$ be Adinkras, with $N_{1}$ and $N_{2}$ the respective number of colors. Let $f_{i}: X_{i} \rightarrow \widehat{\mathbb{C}}$, for $i=1,2$, be the associated Belyi maps. The fibered product $A=A_{1} \times_{\mathcal{I}} A_{2}$, with $\mathcal{I}=[0,1]$, is defined as the graph with set of vertices $V(A)=V_{0}\left(A_{1}\right) \times$ $V_{0}\left(A_{2}\right) \cup V_{1}\left(A_{1}\right) \times V_{1}\left(A_{2}\right)$, where $V_{i}\left(A_{j}\right)$ denotes the vertices of $A_{j}$ that are colored $i$ in the bipartition. The edges are given by all pairs of edges $\left(e_{1}, e_{2}\right) \in E\left(A_{1}\right) \times E\left(A_{2}\right)$ with endpoints in $V(A)$. These are all edges in $E\left(A_{1}\right) \times E\left(A_{2}\right)$, since the $A_{i}$ are bipartite.

The graph obtained in this way has a natural interpretation in terms of the fibered product of the assiciated Riemann surfaces, fibered over the Belyi maps.

Lemma 2.6. Let $A_{N_{i}, k_{i}}$, with $i=1,2$ be two Adinkras, and let $f_{i}: X_{N_{i}, k_{i}} \rightarrow \widehat{\mathbb{C}}$ be the associated Belyi maps. Let $Y$ be the desingularization of the fibered product $\tilde{Y}=X_{N_{1}, k_{1}} \times_{\widehat{\mathbb{C}}}$ $X_{N_{2}, k_{2}}$ fibered along the Belyi maps, with $f: Y \rightarrow \widehat{\mathbb{C}}$ the resulting branched cover. The graph $A=A_{N_{1}, k_{1}} \times_{\mathcal{I}} A_{N_{2}, k_{2}}$ can be identified with the preimage $f^{-1}(\mathcal{I})$ in $Y$.

Proof. The set of vertices $V(A)$ is given by $V(A)=f_{1}^{-1}(0) \times f_{2}^{-1}(0) \cup f_{1}^{-1}(1) \times f_{2}^{-1}(1)$, hence it corresponds to the locus $\left\{(z, w) \in X_{N_{1}, k_{1}} \times X_{N_{2}, k_{2}}: f_{1}(z)=f_{2}(w)=0\right\} \cup\{(z, w) \in$ $\left.X_{N_{1}, k_{1}} \times X_{N_{2}, k_{2}}: f_{1}(z)=f_{2}(w)=1\right\} \subset X_{N_{1}, k_{1}} \times_{\widehat{\mathbb{C}}} X_{N_{2}, k_{2}}$. The edges $E(A)$ can be identified with the set $\left\{(z, w) \in X_{N_{1}, k_{1}} \times X_{N_{2}, k_{2}}: f_{1}(z)=f_{2}(w) \in \mathcal{I}\right\} \subset X_{N_{1}, k_{1}} \times \widehat{\mathbb{C}} X_{N_{2}, k_{2}}$.

Lemma 2.7. The fibered product $A=A_{1} \times_{\mathcal{I}} A_{2}$ is a chromotopology with a ranking $h$ : $V(A) \rightarrow \mathbb{Z}$, which send white/black vertices of the bipartition to even/odd numbers. When the numbers of edge colorings $N_{1}$ and $N_{2}$ are coprime, there is a unique such chromotopology structure, while otherwise there are several inequivalent structures, corresponding to the different choices of colorings. One of these corresponds to the embedding $A=A_{N_{1}, k_{1}} \times_{\mathcal{I}}$ $A_{N_{2}, k_{2}} \simeq f^{-1}(\mathcal{I})$ in $Y$, while the other choices correspond to other surfaces.

Proof. The graph $A$ is bipartite with $V_{0}(A)=V_{0}\left(A_{1}\right) \times V_{0}\left(A_{2}\right)$ and $V_{1}(A)=V_{1}\left(A_{1}\right) \times V_{1}\left(A_{2}\right)$. The edges can be colored by $N=\operatorname{lcm}\left\{N_{1}, N_{2}\right\}$ colors, with an edge $\left(e_{1}, e_{2}\right)$ colored by $\left(c_{1}\left(e_{1}\right), c_{2}\left(e_{2}\right)\right.$, with $c_{i}$ the coloring on $A_{i}$. Note that, when going around a vertex in $X_{1}$, the colors go cyclically from 1 to $N_{1}$, and similarly on $X_{2}$, with colors cyclically ordered from 1 to $N_{2}$. Thus, on the fibered product, the elements $c_{i}$ in the pairs $\left(c_{1}, c_{2}\right)$ have periodicities $N_{i}$. This implies that, when $N_{1}$ and $N_{2}$ are not coprime, the different orbits determine different choices of rainbows of colors, hence different possible resulting chromotopologies. Since the graphs $A_{i}$ are bipartite, the set of edges in $A$ between two vertices $v=\left(v_{1}, v_{2}\right)$ and $w=\left(w_{1}, w_{2}\right)$ consists of the product $E_{v_{1}, v_{2}}\left(A_{1}\right) \times E_{w_{1}, w_{2}}\left(A_{2}\right)$ of the sets of edges in 
$A_{i}$ connecting the two respective vertices. Since the $A_{i}$ have no parallel edges, there is only one edge in each set $E_{v_{1}, v_{2}}\left(A_{1}\right)=\left\{e_{1}\right\}$ and $E_{w_{1}, w_{2}}\left(A_{2}\right)=\left\{e_{2}\right\}$, hence there is a unique $e=\left(e_{1}, e_{2}\right)$ connecting $v=\left(v_{1}, v_{2}\right)$ and $w=\left(w_{1}, w_{2}\right)$ in $A$, with color $c(e)=\left(c_{1}\left(e_{1}\right), c_{2}\left(e_{2}\right)\right)$. Thus, each vertex in $A$ has valence $N$ and is incident to exactly one edge of each color. Given two distinct colors $(i, j) \neq\left(i^{\prime}, j^{\prime}\right)$, consider the set of edges of $A$ with color either $c(e)=(i, j)$ or $c(e)=\left(i^{\prime}, j^{\prime}\right)$. The condition $(i, j) \neq\left(i^{\prime}, j^{\prime}\right)$ occurs when either $i \neq i^{\prime}$ or when $i=i^{\prime}$ and $j \neq j^{\prime}$. In the first case, the set we are considering consists of

$$
\left(c_{1}^{-1}(i) \cup c_{1}^{-1}\left(i^{\prime}\right)\right) \times E\left(A_{2}\right) .
$$

In this case, the left factor consists of a disjoint union of 4-cycles, hence the product set also does, as it is a disjoint union of a copy of such a disjoint union of 4-cycles, for each choice of an element of $E\left(A_{2}\right)$. In the second case, the set we are considering consists of

$$
c_{1}^{-1}(i) \times\left(c_{2}^{-1}(j) \cup c_{2}^{-1}\left(j^{\prime}\right)\right) .
$$

Again the second factor is a disjoint union of 4-cycles, hence the product also is. This shows that the fibered product $A$ is an $N$-chromotopology. To show that it is an Adinkra, we need to check that it is well-dashed and it has a ranking $h: V(A) \rightarrow \mathbb{Z}$ with $h\left(V_{0}(A)\right) \subset 2 \mathbb{Z}$ and $h\left(V_{1}(A)\right) \subset 2 \mathbb{Z}+1$. We obtain a ranking with the desired property by setting $h\left(v_{1}, v_{2}\right)=$ $h_{1}\left(v_{1}\right) \cdot h_{2}\left(v_{2}\right)$, where $h_{i}: V\left(A_{i}\right) \rightarrow \mathbb{Z}$ are the rankings of the Adinkras $A_{i}$. Since $h_{1}\left(v_{1}\right)$ and $h_{2}\left(v_{2}\right)$ are either simultaneously even or simultaneously odd for $\left(v_{1}, v_{2}\right) \in V(A)$, their product is, respectively, even on $V_{0}(A)$ and odd on $V_{1}(A)$.

As an example where the construction above gives rise to different surfaces with different rainbows of colors, consider the case where both $A_{1}$ and $A_{2}$ are isomorphic to the same Adinkra with $N_{1}=N_{2}=8$, and with 16 vertices ( 8 bosons and 8 fermions). Then the construction of Lemma 2.7 above determines 8 different surfaces, each with 8 colors, corresponding to the different choices of $c_{1}-c_{2} \bmod 8$, rather than a single surface with 64 different colors. Indeed, a rainbow of 64 colors would not be compatible with the Adinkra structure, which would require, in that case, at least $2^{32}$ vertices rather than 128 (64 bosons and 64 fermions). (This example was suggested to us by Kevin Iga.)

In the rest of this section, when we refer to "the fibered product chromotopology" we mean the choice of rainbow of colors in Lemma 2.7, such that the resulting chromotopology corresponds to the Riemann surface $Y$ with Belyi map $f: Y \rightarrow \widehat{\mathbb{C}}$, with the embedding $A=A_{N_{1}, k_{1}} \times_{\mathcal{I}} A_{N_{2}, k_{2}} \simeq f^{-1}(\mathcal{I})$.

The dashings $d_{i}$ on the Adinkras $A_{i}$, do not immediately extend to a dashing on the fibered product Adinkra $A=A_{1} \times_{\mathcal{I}} A_{2}$ constructed as above, unlike the case of the Cartesian product structure recalled above, considered in [26]. To see where the problem lies, notice that the 2-colored 4 -cycles in $A$ correspond to choices of two colors $(i, j) \neq\left(i^{\prime}, j^{\prime}\right)$. This corresponds to the cases $i=i^{\prime}$ and $j \neq j^{\prime}$, or $i \neq i^{\prime}$ and $j \neq j^{\prime}$, or $i \neq i^{\prime}$ and $j=j^{\prime}$. In the first two cases the dashing $d_{1}$ would give a dashing on $A$ with the property that each of these 2-colored 4-cycles has an odd number of dashed lines, and similarly the dashing $d_{2}$ would work for the second and third case, but each separately would not cover all cases, as the remaining cases would have an even number of dashings.

However, as shown in [13], using the result of [7] on Kasteleyn orientations and spin curves, the choice of a dashing on an Adinkra $A_{N, k}$ determines a dimer configuration, hence a spin structure on the curve $X_{N, k}$, in such a way that equivalent dashings determine the same spin structure. Thus, it is possible to show that the dashings on the two Adinkras $A_{i}$ 
determine a choice of dashing (up to equilavence) on the fibered product $A=A_{1} \times_{\mathcal{I}} A_{2}$ by showing that a choice of spin structures on the respective Riemann surfaces $X_{i}$ determines uniquely a choice of a spin structure on the resulting Riemann surface $Y$ obtained by desingularizing $\tilde{Y}=X_{1} \times_{\widehat{\mathbb{C}}} X_{2}$.

Lemma 2.8. There is a one-to-one correspondence between pairs of dashings up to vertex change equivalence on the Adinkras $A_{i}$ and dashings on the fibered product chromotopology $A=A_{1} \times_{\mathcal{I}} A_{2}$ up to vertex change equivalence.

Proof. Because of the one-to-one correspondence between dashings up to vertex change equivalence on a chromotopology $A$ and spin structures on the associated Riemann surface $X$ with $A \subset X$, it suffices to show that there is a one-to-one correspondence between pairs $\left(\mathfrak{s}_{1}, \mathfrak{s}_{2}\right)$ of spin structures on $X_{1}$ and $X_{2}$ and spin structures on the fibered product $Y$. It is well known that there are exactly $2^{2 g}$ different spin structures on a Riemann surface of genus $g$. Thus, we only need to verify the relation between the genera $g_{1}=g\left(X_{1}\right)$ and $g_{2}=g\left(X_{2}\right)$ and the genus $g=g(Y)$. Since the base space $\widehat{\mathbb{C}}$ of the fibered product is simply connected, the cohomology of the fibered product $\tilde{Y}=X_{1} \times_{\widehat{\mathbb{C}}} X_{2}$ is computed by the Eilenberg-Moore spectral sequence with $E_{2}^{p, q}=\operatorname{Tor}_{H^{*}(\widehat{\mathbb{C}})}^{p, q}\left(H^{*}\left(X_{1}\right), H^{*}\left(X_{2}\right)\right)$, converging to $H^{*}\left(X_{1} \times_{\widehat{\mathbb{C}}} X_{2}\right)$. This reduces to computing $H^{*}\left(X_{1}\right) \otimes_{H^{*}(\widehat{(} \mathbb{C})} H^{*}\left(X_{2}\right)$ as a tensor product of graded modules over a graded ring. This gives $H_{1}(\tilde{Y}) \simeq \mathbb{Z}^{2\left(g_{1}+g_{2}\right)}$, hence the genera add, $g=g_{1}+g_{2}$.

2.8. M-origami of Adinkras. In the rest of this section we show that Adinkra graphs admit embeddings in origami curves. We use the construction of the Riemann surface $X_{N, k}$ and the Belyi map $f: X_{N, k} \rightarrow \widehat{\mathbb{C}}$ associated to an Adinkra $A_{N, k}$, as in [13], together with the construction of M-origami of [38], which obtains an origami curve $O(f)=(p: Y \rightarrow E)$ from a Belyi map $f: X \rightarrow \widehat{\mathbb{C}}$, to show that the Adinkra graph can be embedded (with a

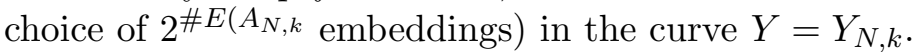

Lemma 2.9. Let $A=A_{N, k}$ be an Adinkra. There exists an origami curve $Y_{N, k}$, with $p: Y_{N, k} \rightarrow E$ ramified only at $\{\infty\}$, determined by the Adinkra, and a collection of $2^{\# E(A)}$ embeddings of the Adinkra $A_{N, k}$ in $Y_{N, k}$.

Proof. Let $X_{N, k}$ be the Riemann surface associated to the Adinkra $A=A_{N, k}$, with Belyi map $f: X_{N, k} \rightarrow \widehat{\mathbb{C}}$. Following the construction of the origami curve $O(f)=\left(p: Y_{N, k} \rightarrow E\right)$ associated to this Belyi map. The origami curve is determined by the Adinkra $A_{N, k}$ because the Riemann surface $X_{N, k}$ and the Belyi map $f: X_{N, k} \rightarrow \widehat{\mathbb{C}}$ are. Let $\Gamma$ denote the graph in $E$ obtained as $h^{-1}\left(f\left(A_{N, k}\right)\right.$, where $h: E \rightarrow \widehat{\mathbb{C}}$ is the double cover ramified at $\{0,1, \infty, \lambda\}$. Since the image $f\left(A_{N, k}\right)$ in $\widehat{\mathbb{C}}$ consists of the interval $\mathcal{I}=[0,1]$, the set of vertices $V(\Gamma)=h^{-1}(0) \cup h^{-1}(1)$ consists of two points, $V(\Gamma)=\{0,1\}$ and the set of edges consists of two parallel edges connecting the two vertices, $E(\Gamma)=\left\{e_{1}, e_{2}\right\}=h^{-1}\left(\mathcal{I}^{o}\right)$, with $\mathcal{I}^{o}=(0,1)$. Consider then the preimage in $\tilde{Y}_{N, k}=E \times_{\widehat{\mathbb{C}}} X_{N, k}$ of the interval $\mathcal{I}$ in $\widehat{\mathbb{C}}$. This consists of a graph $\tilde{A}$ with set of vertices $V(\tilde{A})=V(A)$ and set of edges $E(\tilde{A})=E(A) \cup E(A)$ consisting of two copies of the set of edges of $A$, with each edge of $A$ replaced by a pair of parallel edges. Thus, we obtain in this way $2^{\# E(A)}$ ways of embedding the graph $A=A_{N, k}$ in $\tilde{Y}_{N, k}$ (hence in the desingularization $Y_{N, k}$ ) given by choosing, in all possible ways, one of the two parallel edges. 


\section{Spectral triples from Supersymmetric Algebras}

As shown in [9], in the setting of Noncommutative Geometry, it is possible to encode and generalize the data of compact smooth Riemannian spin manifolds in the form of a triple $(\mathcal{A}, \mathcal{H}, D)$ of an involutive algebra, a Hilbert space on which it is represented by bounded operators, and a self-adjoint Dirac operator on the Hilbert space, with compact resolvent and with bounded commutators with elements of the algebra. In the case of an ordinary compact smooth spin manifold $X$, these data are $\left(\mathcal{C}^{\infty}(X), L^{2}(X, \mathbb{S}), \not D\right)$ with $\mathbb{S}$ the spinor bundle and $\not D$ the Dirac operator on $X$, for a given choice of spin structure. This axiomatization makes it possible to extend methods of Riemannian geometry to spaces that are not ordinary manifolds, including quantum groups, fractals, noncommutative spaces (like noncommutative tori), and the almost-commutative geometries used for the construction of particle physics models. The spectral action functional of a spectral triple $(\mathcal{A}, \mathcal{H}, D)$ is a natural action functional, which corresponds to a theory of gravity on this geometry. In the case of almost-commutative geometries ts large energy asymptotic expansion recovers classical physical action functionals for gravity coupled to matter.

\section{Spectral Action}

The spectral action is a natural construction of an action functional for a spectral triple, defined in terms of the spectrum of the Dirac operator, in the form of a regularized trace $\operatorname{Tr}(f(D / \Lambda)$ ), where $f$ is a suitable test function (a smooth approximation to a cutoff function) and $\Lambda$ is an energy scale. It was originally introduced in [5], and it became an extremely useful tool for particle physics and gravity models based on noncommutative geometry, see for instance [46] for a general survey.

Here we associate to a 1D supersymmetry algebra a spectral triple and a spectral action functional, through the construction of [13] of the Riemann surface $X_{N, k}$ associated to an Adinkra graph $A_{N, k}$. We show that the spectral action can be computed using the Selberg trace formula. We then refine the construction to include the Super Riemann Surface structure on $X_{N, k}$ determined by the Adinkra, and we relate the resulting super spectral action to the Selberg supertrace formula of [1].

We then also discuss the spectral geometry and spectral action associated to the origami curve $O(f)=\left(p: Y_{N, k} \rightarrow E\right)$ associated to the Belyi map $f: X_{N, k} \rightarrow \widehat{\mathbb{C}}$, as another possible construction of a spectral geometry associated to a 1D supersymmetry algebra.

4.1. Selberg trace formula and the Laplacian spectral action. Let $X=\Gamma \backslash \mathbb{H}$ be a compact hyperbolic Riemann surfaces of genus $g=g(X) \geq 2$, uniformized by a Fuchsian group $\Gamma \subset \mathrm{SL}_{2}(\mathbb{R})$, endowed with the hyperbolic metric of constant curvature -1 induced from the hyperbolic upper half place $\mathbb{H}$, with $\Gamma$ acting by isometries.

Definition 4.1. Let $\left\{\lambda_{j}=\frac{1}{4}+r_{j}^{2}\right\}_{j \in \mathbb{Z}_{+}}$be the spectrum of the Laplacian $\Delta$ in the hyperbolic metric of constant curvature -1 on $X$. For $f \in \mathcal{S}(\mathbb{R})$ a rapidly decaying even test function, the Laplacian spectral action on $X$ is given by

$$
\mathcal{S}_{\Delta, f}(\Lambda):=\sum_{j=0}^{\infty} f\left(r_{j} / \Lambda\right) .
$$


Let $\mathcal{G}_{X}$ denote the set of oriented closed geodesics on $X$. For an oriented closed geodesic $\gamma \in \mathcal{G}_{X}$ let $\ell(\gamma)$ denote the length and $N_{\gamma}=\exp (\ell(\gamma))$ the norm. Moreover, let $\lambda(\gamma):=\ell\left(\gamma_{0}\right)$ where $\gamma_{0}$ is the unique oriented primitive closed geodesic, such that $\gamma=\gamma_{0}^{m}$ for some $m \in \mathbb{N}$. The Laplacian spectral action can be computed via the Selberg trace formula.

Lemma 4.2. Let $h \in \mathcal{C}^{\infty}(\mathbb{R})$ be an even compactly supported test function. We assume that $\operatorname{supp}(h)=[-1,1]$. Let $f \in \mathcal{S}(\mathbb{R})$ be the rapidly decaying even test function obtained as Fourier transform $f=\hat{h}$. The Laplacian spectral action on $X$ satisfies

$$
\mathcal{S}_{\Delta, f}(\Lambda)=\Lambda^{2}(g(X)-1) \int_{0}^{\infty} r f(r) \tanh (\Lambda \pi r) d r+\Lambda \sum_{\gamma \in \mathcal{G}_{X, \Lambda}} \frac{\lambda(\gamma)}{N_{\gamma}^{1 / 2}-N_{\gamma}^{-1 / 2}} h\left(\Lambda \log N_{\gamma}\right),
$$

where the sum on the right hand side is over the set of oriented closed geodesics

$$
\mathcal{G}_{X, \Lambda}=\left\{\gamma \in \mathcal{G}_{X}: \ell(\gamma) \leq \frac{1}{\Lambda}\right\}
$$

Proof. The Selberg trace formula gives

$$
\sum_{j=0}^{\infty} f\left(r_{j}\right)=\frac{A(X)}{4 \pi} \int_{0}^{\infty} r f(r) \tanh (\pi r) d r+\sum_{\gamma \in \mathcal{G}_{X}} \frac{\lambda(\gamma)}{N_{\gamma}^{1 / 2}-N_{\gamma}^{-1 / 2}} h\left(\log N_{\gamma}\right),
$$

where $A(X)$ is the area of the surface $X$, which by Gauss-Bonnet satisfies $A(X)=4 \pi(g-1)$, with $g=g(X) \geq 2$ the genus of $X$. The expression 4.2 is then an immediate consequence of (4.4), after replacing $f(r)$ with $f_{\Lambda}(r)=f(r / \Lambda)$ and $h(s)$ with $h_{\Lambda}(s)=\Lambda h(\Lambda s)$, so that $f_{\Lambda}=\hat{h}_{\Lambda}$. Since the function $h$ has support the interval $[-1,1]$, the function $h_{\Lambda}$ has support $[-1 / \Lambda, 1 / \Lambda]$, hence only the geodesics $\gamma$ in the set $\mathcal{G}_{X, \Lambda}$ contribute to the sum.

Corollary 4.3. Let $\mathcal{C}_{\Gamma}$ denote the set of conjugacy classes of primitive simple hyperbolic elements in the Fuchsian group $\Gamma$. For $P \in \mathcal{C}_{\Gamma}$, let $t_{P}$ denote the translation length of an element of $P$. The Laplacian spectral action on the hyperbolic surface $X=\Gamma \backslash \mathbb{H}$ satisfies

$$
\begin{aligned}
\mathcal{S}_{\Delta, f}(\Lambda) & =\Lambda^{2}(g(X)-1) \int_{0}^{\infty} r f(r) \tanh (\Lambda \pi r) d r \\
& +\Lambda \sum_{P \in \mathcal{C}_{\Gamma}} \operatorname{arccosh}\left(\frac{t_{P}}{2}\right) \sum_{\ell \in S_{\Lambda}(P)} \frac{h\left(\Lambda 2 \ell \operatorname{arccosh}\left(\frac{t_{P}}{2}\right)\right)}{\sinh \left(\ell \operatorname{arccosh}\left(\frac{t_{P}}{2}\right)\right)},
\end{aligned}
$$

where, for a given $P \in \mathcal{C}_{\Gamma}$, the sum is over the finite set

$$
S_{\Lambda}(P)=\left\{\ell \in \mathbb{N}: 2 \ell \operatorname{arccosh}\left(t_{P} / 2\right) \leq 1 / \Lambda\right\} .
$$

Proof. Since all elements in $\Gamma \backslash\{1\}$ are hyperbolic, it is well known that the Selberg trace formula 4.4 can be rewritten equivalently in terms of conjugacy classes $\mathcal{C}_{\Gamma}$ as

$$
\begin{aligned}
\sum_{j=0}^{\infty} f\left(r_{j}\right) & =(g(X)-1) \int_{0}^{\infty} r f(r) \tanh (\pi r) d r \\
& +\sum_{P \in \mathcal{C}_{\Gamma}} \operatorname{arccosh}\left(\frac{t_{P}}{2}\right) \sum_{\ell=1}^{\infty} \frac{h\left(2 \ell \operatorname{arccosh}\left(\frac{t_{P}}{2}\right)\right)}{\sinh \left(\ell \operatorname{arccosh}\left(\frac{t_{P}}{2}\right)\right)} .
\end{aligned}
$$

Replacing $f(r)$ with $f_{\Lambda}(r)=f(r / \Lambda)$ and $h(s)$ with $h_{\Lambda}(s)=\Lambda h(\Lambda s)$, we obtain 4.5). Since the function $h$ has support $[-1,1]$, for a given $P \in \mathcal{C}_{\Gamma}$, the only integers $\ell$ contributing to the sum are those in the set $S_{\Lambda}(P)$. 
4.2. The Dirac spectral action on a compact Riemann surface. In noncommutative geometry, instead of working with the Laplacian $\Delta$ and the Laplace spectral action discussed above, one considers the Dirac operator $D$ and the Dirac Laplacian $D^{2}$. Indeed, Dirac operators are abstracted to the more general setting of spectral triples $(\mathcal{A}, \mathcal{H}, D)$, which generalize to possibly noncommutative settings the spin geometry $\left(\mathcal{C}^{\infty}(X), L^{2}(X, \mathbb{S}), D\right)$, with $\mathbb{S}$ the spinor bundle of a compact Riemannian spin manifold $X$, see 9 for more details. The spectral action functional, for a Dirac operator $D$ of a spectral triple, is defined (see $[5])$, for $f \in \mathcal{S}(\mathbb{R})$ an even rapidly decaying test function, as

$$
\mathcal{S}_{D, f}(\Lambda):=\operatorname{Tr}(f(D / \Lambda))
$$

In the case of a compact Riemann surface $X$ of genus $g \geq 2$, with the constant negative curvature hyperbolic metric, the behavior of the spectral action functional (4.8) is similar to the behavior of the Laplacian spectral action discussed in the previous subsection. The effect on the Selberg trace formula of replacing the Laplacian $\Delta$ by the Dirac Laplacian $D^{2}$ is discussed in [4]. When we adapt these results to the argument given in the previous subsection, we obtain the following result for the spectral action of a hyperbolic surface.

In the case of the Dirac operator, the choice of the spin structure on the Riemann surface $X$ is encoded in the choice of the spinor bundle $\mathbb{S}$ over $X$. A vector bundle on $X$ corresponds to a trivial pullback bundle on the universal cover, $\pi^{*}(\mathbb{S})=\mathbb{H} \times S$, with $S$ the fiber of $\mathbb{S}$, together with the datum of a transition function $\sigma: \mathbb{H} \times \Gamma \rightarrow \operatorname{GL}(S)$, where $\Gamma \subset \mathrm{PSL}_{2}(\mathbb{R})$ is the uniformizing Fuchsian group, so that spinor sections of $\mathbb{S}$ are identified with functions $\psi: \mathbb{H} \rightarrow S$ with $\psi(\gamma z)=\sigma(z, \gamma) \psi(z)$. The transition function $\sigma$ can be encoded by the pair of an automorphy factor $j: \mathbb{H} \times \tilde{\Gamma} \rightarrow \operatorname{GL}(S)$ and a character $\chi: \tilde{\Gamma} \rightarrow U(1)$, where $\tilde{\Gamma} \subset \mathrm{SL}_{2}(\mathbb{R})$ is such that $\Gamma=\tilde{\Gamma} /\{ \pm 1\}$, with $\chi(-1)=-1$, see $\S$ III of [4] and [42, so that the spinor sections satisfy the automorphic condition $\Psi(\gamma z)=\chi(\gamma) j(z, \gamma) \Psi(z)$, where $\Psi(z)=\left(\psi_{1}(z), \psi_{2}(z)\right)$ and $j(z, \gamma)$ diagonal with entries $j(z, \gamma, \pm 1)$. (We consider here only the case of the Dirac operator $D=D_{1}$ of [4] with weight $k=1$, that is, the standard Dirac operator.) The matching between the eigenfunctions $\Psi=\left(\psi_{1}, \psi_{2}\right)$ of $D$ with eigenvalue $\lambda$ and the eigenfunctions $\psi=\psi_{1}$ of the Laplacian with eigenvalue $\lambda^{2}+1 / 4$ is proved in Proposition 1 of [4].

In the specific case where the Riemann surface $X$ is defined over a number field, so that it admits a Belyi map $f: X=H \backslash \mathbb{H} \rightarrow \widehat{\mathbb{C}}=\Delta_{p, q, r} \backslash \mathbb{H}$ and a uniformization $X=H \backslash \mathbb{H}$ by a finite index subgroup of a triangle group $\Delta_{p, q, r}$, as in [8], the automorphic functions approach of [4] to the spectral decomposition of the Dirac operator can be made more explicit, using the results of [14] on automorphic forms for triangle groups.

Lemma 4.4. Let $X$ be a compact Riemann surface of genus $g=g(X) \geq 2$, endowed with the hyperbolic metric of constant curvature -1 . Let $\mathfrak{s}$ be a spin structure on $X$ and $D=D_{\mathfrak{s}}$ the corresponding Dirac operator, acting on sections of the spinor bundle $\mathbb{S}=\mathbb{S}_{\mathfrak{s}}$ on $X$. Let $\chi: \tilde{\Gamma} \rightarrow U(1)$ be the character determined by the spin structure $\mathfrak{s}$, as above. Let $h \in \mathcal{C}^{\infty}(\mathbb{R})$ be an even compactly supported test function with support $\operatorname{supp}(h)=[-1,1]$ and let $f \in \mathcal{S}(\mathbb{R})$ be the Fourier transform $f=\hat{h}$. The Dirac spectral action then satisfies

$$
\begin{aligned}
\mathcal{S}_{D, f}(\Lambda) & =\Lambda^{2}(g(X)-1) \int_{\mathbb{R}} r f(r) \operatorname{coth}(\pi r) d r \\
& +\Lambda \sum_{P \in \mathcal{C}_{\bar{\Gamma}}} \sum_{\ell=1}^{\infty} \frac{\chi\left(P^{\ell}\right) \operatorname{arccosh}\left(\frac{t_{P}}{2}\right) h\left(\Lambda 2 \ell \operatorname{arccosh}\left(\frac{t_{P}}{2}\right)\right)}{\sinh \left(\ell \operatorname{arccosh}\left(\frac{t_{P}}{2}\right)\right)}
\end{aligned}
$$


where $\mathcal{C}_{\bar{\Gamma}}$ is the set of $\bar{\Gamma}$-conjugacy classes.

Proof. According to Theorem 1 of [4], the Selberg trace formula for the Dirac operator on a hyperbolic compact Riemann surface is obtained by modifying the case (4.7) of the Laplacian in the following way:

$$
\begin{aligned}
\sum_{j=0}^{\infty} f\left(\lambda_{j}\right) & =(g(X)-1) \int_{\mathbb{R}} r f(r) \operatorname{coth}(\pi r) d r \\
& +\sum_{P \in \mathcal{C}_{\bar{\Gamma}}} \operatorname{arccosh}\left(\frac{t_{P}}{2}\right) \sum_{\ell=1}^{\infty} \chi\left(P^{\ell}\right) \frac{h\left(2 \ell \operatorname{arccosh}\left(\frac{t_{P}}{2}\right)\right)}{\sinh \left(\ell \operatorname{arccosh}\left(\frac{t_{P}}{2}\right)\right)},
\end{aligned}
$$

where $\left\{\lambda_{j}\right\}=\operatorname{Spec}\left(D_{\mathfrak{s}}\right)$. Again, we replace $f(r)$ with $f_{\Lambda}(r)=f(r / \Lambda)$ and $h(s)$ with $h_{\Lambda}(s)=\Lambda h(\Lambda s)$, to obtain 4.9 .

4.3. Selberg zeta function and the Spectral Action. An approach to the Selberg trace formula via the Selberg zeta function is described in [16], see also §VII of [4] for the Dirac case. For $\Re(s)>1$ and $\Re(\sigma)>1$, the trace formula applied to the test function

$$
f(\lambda)=\left(\lambda^{2}+\left(s-\frac{1}{2}\right)^{2}\right)^{-1}-\left(\lambda^{2}+\left(\sigma-\frac{1}{2}\right)^{2}\right)^{-1}
$$

gives as the second term in the right-hand-side of (4.10) the expression

$$
\frac{1}{2 s-1} \frac{Z_{\Gamma}^{\prime}(s)}{Z_{\Gamma}(s)}-\frac{1}{2 \sigma-1} \frac{Z_{\Gamma}^{\prime}(\sigma)}{Z_{\Gamma}(\sigma)},
$$

where $Z_{\Gamma}(s)$ is the Selberg zeta function

$$
Z_{\Gamma}(s)=\prod_{P \in \mathcal{C}_{\bar{\Gamma}}} \prod_{\ell}\left(1-\chi(P) e^{L_{P}(s+\ell)}\right),
$$

where the set $\mathcal{C}_{\bar{\Gamma}}$ is identified with the set of primitive closed geodesics on $X=\Gamma \backslash \mathbb{H}$ and $L_{P}=2 \operatorname{arccosh}\left(\frac{t_{P}}{2}\right)$ with the geodesic length.

The Selberg zeta function can sometimes be computed in terms of a coding of geodesics via symbolic dynamics and the Fredholm determinant of an associated Ruelle transfer operators. For the case of the modular group $\mathrm{SL}_{2}(\mathbb{Z})$ and finite index subgroups, see [6]. Similar results have been obtained for a particular class of Fuchsian triangle groups, the Hecke triangle groups, see [37], [45], and generalized to an algorithm for symbolic coding of geodesics applicable to other Fuchsian groups in [41.

Hecke triangle groups $H_{q}$ are the Fuchsian triangle groups of the form $\Delta_{2, q, \infty}$. They have a presentation $H_{q}=\left\langle S, T_{q} \mid S^{2}=\left(S T_{q}\right)^{q}=1\right\rangle$, with $S z=-1 / z$ and $T_{q} z=z+\lambda_{q}$, where $\lambda_{q}=2 \cos (\pi / q)$. A compact Riemann surface $X$ that admits a regular tessellation by hyperbolic $q$-gons also admits a uniformization by a finite index subgroup $H$ of a Hecke group $H_{q}$, with a Belyi map given by the projection $f: H \backslash \mathbb{H} \rightarrow \mathbb{P}^{1}=H_{q} \backslash \mathbb{H}$, with the single cusp $\{\infty\}$ of $H_{q}$ added in the compactification $\mathbb{P}^{1}$.

The Selberg zeta function of a Hecke triangle group $H_{q}=\Delta_{2, q, \infty}$ can be computed explicitly, in terms of thermodynamic formalism and the appropriate transfer operator, associated to a continued fraction algorithm, as shown in [37, [45]. More precisely, the 
Selberg zeta function is obtained, in the case of the Hecke triangle groups $H_{q}$ as a ratio of Fredholm determinants

$$
Z_{H_{q}}(s)=\frac{\operatorname{det}\left(1-\mathcal{L}_{s}\right)}{\operatorname{det}\left(1-\mathcal{K}_{s}\right)}
$$

where $\mathcal{L}_{s}$ is the Liouville transfer operator of the continued fraction algorithm of $H_{q}$, in analogy to the case of $\mathrm{SL}_{2}(\mathbb{Z})$ of $\left[6\right.$. However, the operator $\mathcal{L}_{s}$ alone introduces an overcounting, in the case of triangle groups $H_{q}$, which is corrected by another transfer operator $\mathcal{K}_{s}$. Moreover, in [39] it is shown that the Maass cusp forms of Hecke triangle groups $H_{q}$ are solutions of a certain functional equation, generalizing the one of Lewis-Zagier for the modular group, which characterizes fixed points of the transfer operator associated to the Selberg zeta function.

A coding of geodesics for the more general triangle groups $\Delta_{p, q, \infty}$ and associated transfer operators, whose Fredholm determinant is similarly related to the Selberg zeta function, were obtained in [17]. A more general approach to symbolic dynamics and transfer operators, for arbitrary 2-dimensional hyperbolic good orbifolds $X$, is developed in [41.

These methods provide an approach to compute the spectral action $\mathcal{S}_{D, f}(\Lambda)$ with a test function of the form (4.11) using the Selberg zeta function computed via the transfer operator method.

The transfer operator method in general consists of a construction of a cross section for the geodesic flow on the hyperbolic surface $X=\Gamma \backslash \mathbb{H}$, where $\Gamma$ is a cocompact Fuchsian group, using a choice of fundamental domains, so that the first return map determines a discrete dynamical system. This dynamical system is then encoded on the boundary $\mathbb{P}^{1}(\mathbb{R})$ of $\mathbb{H}$ in terms of a family of finitely many local diffeomorphisms, determined by a coding of geodesics by infinite sequences in an alphabet $\Sigma$ in which the first return map becomes the shift map of the symbolic dynamics. Given the resulting map $F$ describing this boundary dynamics, the associated transfer operator (depending on a parameter $\beta \in \mathbb{C}$ ) is given by

$$
\mathcal{L}_{F, \beta} f(x)=\sum_{y \in F^{-1}(x)}\left|F^{\prime}(y)\right|^{-\beta} f(y) .
$$

We refer the reader to [41] for a detailed construction of the transfer operator for a class of Fuchsian groups.

4.4. Dirac spectral action of Adinkras. Given an Adinkra chromotopology $A_{N, k}$ and the associated Riemann surface $X_{N, k}$ with a Belyi map $f: X_{N, k} \rightarrow \hat{\mathbb{C}}$ as in [13], we can consider the spectral triple $\left(\mathcal{C}^{\infty}\left(X_{N, k}\right), L^{2}\left(X_{N, k}, \mathbb{S}_{\mathfrak{s}}\right), D\right)$ with $D$ the Dirac operator $D=D_{\mathfrak{s}}$ associated to the spin structure $\mathfrak{s}$ determined by the dashing of the Adinkra graph as in [13]. By the discussion above and the results of [8], we know that the Riemann surface can be uniformized as $X_{N, k}=H \backslash \mathbb{H}$, where $H$ is a finite index subgroup of the Fuchsian triangle group $\Delta_{N, N, 2}$. While in general it is difficult to describe the subgroup $H$ explicitly, it is still possible to use the method of [41], applied to the Fuchsian triangle group $\Delta_{N, N, 2}$, as an approach to the computation of the spectral action, by including the finite coset space $\Delta_{N, N, 2} / H$ in the construction of the transfer operator, as was done for the finite index subgroups of the modular group in [6], see also [32], [33].

More precisely, suppose given a construction as above of a transfer operator $\mathcal{L}_{F, \beta}=\mathcal{L}_{F, \beta, \Gamma}$ based on the coding of the geodesic flow on a hyperbolic surface $X=\Gamma \backslash \mathbb{H}$, for a given Fuchsian group $\Gamma$. There is a way to obtain from it a transfer operator for an arbitrary 
finite index subgroup $H \subset \Gamma$, using the same boundary dynamics that determines $\mathcal{L}_{F, \beta, \Gamma}$. This is a simple generalization of the same construction used in [6], 32, 33] for the case of $\Gamma=\mathrm{SL}_{2}(\mathbb{Z})$. We assume the following general condition: the transfer operator can be written in terms of local determinations of the function $F$ as

$$
\mathcal{L}_{F, \beta, \Gamma} f(x)=\sum_{E} \sum_{s \in \Sigma_{E}} \chi_{E_{s}}(x)\left|g_{s}^{\prime}(x)\right|^{-\beta} f\left(g_{s} x\right),
$$

where $F^{-1}(x)=\cup E$ is a union of pairwise disjoint sets $E=\cup_{s} E_{s}$, labelled by elements $s \in \Sigma_{E} \subset \Sigma$ in the alphabet of the symbolic coding, such that $\left.F\right|_{E}(y)=g_{s}^{-1} y=x$, for elements $g_{s} \in \Gamma$.

Lemma 4.5. Let $\Gamma$ be a Fuchsian group that has a transfer operator $\mathcal{L}_{F, \beta, \Gamma}$, with $F(x)$ the boundary dynamical system providing the coding of geodesics on $\Gamma \backslash \mathbb{H}$, which satisfies (4.15). Let $H \subset \Gamma$ be a finite index subgroup and $X=H \backslash \mathbb{H}$ the corresponding hyperbolic surface. For $P=\Gamma / H$, a coding map for the geodesics on $X$ can be obtained by extending $F(x)$ uniquely to a function $F(x, a)$ with $a \in P$, with transfer operator of the form

$$
\mathcal{L}_{F, \beta, H \subset \Gamma} f(x, a)=\sum_{E} \sum_{s \in \Sigma_{E}} \chi_{E_{s}}(x)\left|g_{s}^{\prime}(x)\right|^{-\beta} f\left(g_{s} a, g_{s} a\right) .
$$

Proof. Let $P=\Gamma / H$ be the coset space, with the left transitive action of $\Gamma$. We extend the map $F(x)$ of the boundary dynamics to a map $F(x, a)$, with $a \in P$, by setting

$$
\left.F\right|_{E_{s} \times P}(x, a)=\left(g_{s}^{-1} x, g_{s}^{-1} a\right) .
$$

Correspondingly we obtain a transfer operator of the form (4.16). This transfer operator and the map $F$ considered here provide a coding of geodesics on $X=H \backslash \mathbb{H}$ through the identification of this quotient with the quotient $\Gamma \backslash(\mathbb{H} \times P)$.

4.5. Supersymmetric Riemann Surfaces. A Super Riemann Surface $M$ is locally modeled on $\mathbb{C}^{1 \mid 1}$, with local coordinates $z$ (bosonic) and theta (fermionic). A non-integrable subbundle $\mathcal{D} \subset T \mathbb{C}^{1 \mid 1}$ is determined by

$$
D_{\theta}=\partial_{\theta}+\theta \partial_{z}
$$

which satisfies

$$
\left[D_{\theta}, D_{\theta}\right]=2 \partial_{z},
$$

so that one has $\mathcal{D} \otimes \mathcal{D} \simeq T M / \mathcal{D}$. We refer the reader to [30], 31] for a detailed treatment of the theory of supermanifolds and in particular Super Riemann Surfaces.

It is shown in [13] that an odd dashing on an Adinkra $A_{N, k}$ determines a Super Riemann Surface structure on $X_{N, k}$.

Thus, one can refine the data of the spectral triple and spectral action discussed above, based on the Riemann surface $X_{N, k}$, by including also the structure of Super Riemann Surface. To this purpose, we modify the definition and computation of the spectral action given above to incorporate the supermanifold structure, by replacing the Selberg trace formula with a Selberg supertrace formula. 
4.6. The Supersymmetric Selberg trace formula. A Selberg super trace formula for Super Riemann Surfaces, based on the Dirac Laplacian, was obtained in [1. Additional results on the Selberg super zeta function were obtained in [20], see also [21]. We consider here the Dirac Laplacian $\Delta=2 Y D \bar{D}$ (the case $m=0$ of the family of Dirac Laplacians considered in [1], [20]), where $-\left(4 Y^{2}\right)^{-1}$ is the superdeterminant of the metric tensor on the super upper half plane $\mathcal{S} \mathbb{H}$, and $D=D_{\theta}=\theta \partial_{z}+\partial_{\theta}$ and $\bar{D}=D_{\bar{\theta}}=-\partial_{\bar{\theta}}+\bar{\theta} \partial_{z}$. Let $\left\{\lambda_{j}^{B}=i r_{j}^{B}+1 / 2\right\}$ and $\left\{\lambda_{j}^{F}=i r_{j}^{F}+1 / 2\right\}$ denote, respectively, the bosonic and fermionic spectra of $\Delta$.

Note that the operator $D$ satisfies $D^{2}=\partial_{z}$, so it can be viewed as a square root of $\partial_{z}$. This is reflected in the structure of the spectrum, with respect to the Dirac spectrum on an ordinary Riemann surface. Thus, a natural type of spectral action functional to consider in this supersymmetric spectrum is obtained by replacing the ordinary Dirac operator by the supersymmetric Dirac Laplacian $\Delta$ and the trace by a supertrace.

Let $f$ be a test function with the properties that $f(i x+1 / 2)$ is in $\mathcal{C}^{\infty}(\mathbb{R})$ with $f(i x+1 / 2) \sim$ $O\left(x^{-2}\right)$ for $x \rightarrow \pm \infty$, and with $f(i z+1 / 2)$ holomorphic for $|\Im(z)| \leq 1+\epsilon$.

Definition 4.6. For a test function $f$ as above. The supersymmetric spectral action of the Super Riemann Surface $\mathcal{S} X=\Gamma \backslash \mathcal{S} \mathbb{H}$ is given by

$$
\mathcal{S}_{\mathcal{S} X, \Delta, f}(\Lambda)=\operatorname{Tr}_{s}(f(\Delta / \Lambda))=\sum_{j=0}^{\infty}\left(f\left(\frac{\lambda_{j}^{B}}{\Lambda}\right)-f\left(\frac{\lambda_{j}^{F}}{\Lambda}\right)\right) .
$$

One can consider also a slightly different version of the supersymmetric spectral action, defined by analogy with the Laplacian spectral action discussed above. Let $\tilde{f}(r)=f(i r+$ $1 / 2)$ with $f$ a test function as above and define the supersymmetric spectral action as

$$
\mathcal{S}_{\mathcal{S} X, \Delta, \tilde{f}}(\Lambda)=\sum_{j=0}^{\infty}\left(\tilde{f}\left(\frac{r_{j}^{B}}{\Lambda}\right)-\tilde{f}\left(\frac{r_{j}^{F}}{\Lambda}\right)\right) .
$$

The difference with respect to the previous version lies in rescaling $r_{j}^{B} \mapsto \Lambda^{-1} r_{j}^{B}$ and $r_{j}^{F} \mapsto \Lambda^{-1} r_{j}^{F}$ rather than $\lambda_{j}^{B}=r_{j}^{B}+1 / 2 \mapsto \Lambda^{-1} \lambda_{j}^{B}=\frac{r_{j}^{B}+1 / 2}{\Lambda}$ and similarly $\lambda_{j}^{F} \mapsto \Lambda^{-1} \lambda_{j}^{F}$.

Let $h$ denote the Fourier transform

$$
h(t)=\frac{1}{2 \pi} \int_{\mathbb{R}} e^{-i t x} f(i x+1 / 2) d x,
$$

of a test function $f$ chosen as above. Let $G(x, \chi)$ be the function

$$
G(x, \chi)=h(x)+h(-x)-\left(\chi e^{-x / 2} h(x)+\chi e^{x / 2} h(-x)\right)
$$

The supersymmetric spectral action can then be computed in terms of the Selberg supertrace formula.

Proposition 4.7. Let $f$ be a test function as above. The supersymmetric spectral action satisfies

$$
\begin{aligned}
\mathcal{S}_{\mathcal{S} X, \Delta, f}(\Lambda) & =i \Lambda(g(X)-1) \int_{\mathbb{R}} f(i r+1 / 2) \tanh (\Lambda \pi r) d r \\
& +\sum_{\gamma \in \mathcal{C}(\Gamma)} \sum_{k=1}^{\infty} \frac{\lambda(\gamma)}{N_{\gamma}^{1 / 2}-N_{\gamma}^{-1 / 2}} G_{\Lambda}\left(\log N_{\gamma}, \chi(\gamma)\right),
\end{aligned}
$$


where $G_{\Lambda}(x, \chi)=h_{\Lambda}(x)+h_{\Lambda}(-x)-\left(\chi e^{-x / 2} h_{\Lambda}(x)+\chi e^{x / 2} h_{\Lambda}(-x)\right)$, with the function $h_{\Lambda}(t)=\Lambda e^{-\frac{t}{2}(\Lambda-1)} h(\Lambda t)$.

Proof. The Selberg supertrace formula is given by ([1], [20])

$$
\begin{aligned}
\sum_{j=0}^{\infty}\left(f\left(\lambda_{j}^{B}\right)-f\left(\lambda_{j}^{F}\right)\right) & =i(g-1) \int_{R} f(i r+1 / 2) \tanh (\pi r) d r \\
& +\sum_{\gamma \in \mathcal{C}(\Gamma)} \sum_{k=1}^{\infty} \frac{\lambda(\gamma)}{N_{\gamma}^{1 / 2}-N_{\gamma}^{-1 / 2}} G\left(\log N_{\gamma}, \chi(\gamma)\right),
\end{aligned}
$$

where the function $G(x, \chi)$ is given by 4.19$)$. Here we identify the set $\mathcal{C}(\Gamma)$ of conjugacy classes of $\Gamma$ with the oriented primitive closed geodesics and we write $\lambda(\gamma)=\ell\left(\gamma_{0}\right)=\log N_{\gamma_{0}}$ for the length of the unique element in the class of $\gamma$ such that $\gamma=\gamma_{0}^{m}$ for some $m \in \mathbb{N}$. We write $N_{\gamma}=\exp (\ell(\gamma))$ for the exponentiated lengths. When scaling the spectrum of the Dirac Laplacian by $\Delta \mapsto \Delta / \Lambda$, we replace the test function $f$ with the scaled function $f_{\Lambda}(\lambda)=$ $f(\lambda / \Lambda)$. Correspondingly, the Fourier transform $h_{\Lambda}(t)=(2 \pi)^{-1} \int_{\mathbb{R}} e^{-i t x} f_{\Lambda}(i x+1 / 2) d x$ is given by $h_{\Lambda}(t)=\Lambda e^{-\frac{t}{2}(\Lambda-1)} h(\Lambda t)$.

The case of the form (4.18) of the supersymmetric spectral action is similar.

Corollary 4.8. Let $f$ be a test function as above. The supersymmetric spectral action in the form 4.18) satisfies

$$
\begin{aligned}
\mathcal{S}_{\mathcal{S} X, \Delta, \tilde{f}}(\Lambda) & =i \Lambda(g(X)-1) \int_{R} \tilde{f}(r) \tanh (\Lambda \pi r) d r \\
& +\Lambda \sum_{\gamma \in \mathcal{C}(\Gamma)} \sum_{k=1}^{\infty} \frac{\lambda(\gamma)}{N_{\gamma}^{1 / 2}-N_{\gamma}^{-1 / 2}} G\left(\Lambda \log N_{\gamma}, \chi(\gamma)\right)
\end{aligned}
$$

Proof. The argument is analogous to the previous case, with $\tilde{f}_{\Lambda}(r)=\tilde{f}\left(\frac{r}{\Lambda}\right)$ and the Fourier transform $h_{\Lambda}(t)=(2 \pi)^{-1} \int_{\mathbb{R}} e^{-i t x} \tilde{f}_{\Lambda}(x) d x=\Lambda h(\Lambda t)$.

4.7. Origami curves and the Laplace spectrum. In addition to the spectral geometry considered above, obtained from the spectrum of the Dirac operator on the (Super) Riemann surface $X_{N, k}$, we can also consider another geometry associated to the Adinkra $A_{N, k}$, namely the origami curve $Y_{N, k}$ considered in Lemma 2.9 .

In the case of a branched cover $p: Y \rightarrow E$ of an elliptic curve $E$, it is shown in [35, [36] that it is possible to construct an infinite set of eigenvalues and eigenfunctions of the Laplacian on $Y$, which we recall briefly here.

Let $\left\{\omega_{k}\right\}_{k=1}^{g}$ be a basis of holomorphic differentials for $Y$ and let $\Omega$ be the period matrix $\Omega_{j k}=\int_{\beta_{j}} \omega_{k}$, with the normalization $\int_{\alpha_{j}} \omega_{k}=\delta_{j k}$ for $\left\{\alpha_{j}, \beta_{j}\right\}$ a symplectic basis of $H_{1}(Y, \mathbb{Z})$. It is shown in [35], 36] that each solution of the equations

$$
m_{i}-\sum_{k=1}^{g} \Omega_{i k} n_{k}=N_{i j}\left(m_{j}-\sum_{k=1}^{g} \Omega_{j k} n_{k}\right)
$$

for $(n, m) \in \mathbb{Z}^{2 g}$, with $N_{i j} N_{j k}=N_{i k}$ determined by $g-1$ elements, determines a metric $g=g^{(n, m)}$ on $Y$. 
One considers then the set of solutions $\left(n^{\prime}, m^{\prime}\right) \in \mathbb{Z}^{2 g}$ of $\omega_{n^{\prime}, m^{\prime}}=c \omega_{n, m}$, for some $c=$ $c\left(n, m, n^{\prime}, m^{\prime}\right)$, where the $\omega_{n, m}$ are the primitive differentials

$$
\begin{gathered}
\omega_{n, m}=\sum_{k=1}^{g} c_{n, m ; k} \omega_{k}, \\
c_{n, m ; k}=\pi \sum_{j=1}^{g} \frac{m_{j}-\sum_{\ell=1}^{g} \bar{\Omega}_{\ell j} n_{\ell}}{\left(\Im(\Omega)^{-1}\right)_{j k}} .
\end{gathered}
$$

As in [36], we denote by $\mathcal{S}_{n, m}(\Omega)$ this set of solutions. This set of solutions in particular includes the $(k n, k m)$ for $k \in \mathbb{Z}$, but can in general be larger. Each such solution $\left(n^{\prime}, m^{\prime}\right)$ determines an eigenvalue

$$
\lambda_{n^{\prime} m^{\prime}}=2 A_{n, m}\left|\frac{m_{i}^{\prime}-\sum_{k=1}^{g} \bar{\Omega}_{i k} n_{k}^{\prime}}{m_{i}-\sum_{k=1}^{g} \bar{\Omega}_{i k} n_{k}}\right|^{2},
$$

of the Laplacian $\Delta=\Delta_{g(n, m)}$, where $A_{n, m}=\frac{i}{4} \int_{Y} \omega_{n, m} \wedge \bar{\omega}_{n, m}$.

Solutions to the equation 4.23 can be seen as a set of consistency equations satisfied by the period matrix of $Y$. It is shown in [36] that a Riemann surface $Y$ has period matrix satisfying these conditions if and only if it is a branched cover of an elliptic curve. Thus, in particular, the construction works for all origami curves.

Thus, given the origami curve $Y=Y_{N, k}$, associated to an Adinkra graph $A_{N, k}$ as in Lemma 2.9, one can consider the spectral action functional associated to the spectrum $\left\{\lambda_{n^{\prime}, m^{\prime}}\right\}$ constructed in [35], 36]. Since the spectrum considered in the action functional should correspond to a square root of the Laplacian, we consider the sequence

$$
\rho_{n^{\prime}, m^{\prime}}= \pm \sqrt{2 A_{n, m}}\left|\frac{m_{i}^{\prime}-\sum_{k=1}^{g} \bar{\Omega}_{i k} n_{k}^{\prime}}{m_{i}-\sum_{k=1}^{g} \bar{\Omega}_{i k} n_{k}}\right|
$$

for $\left(n^{\prime}, m^{\prime}\right) \in \mathcal{S}_{n, m}(\Omega)$, and we define

$$
\mathcal{S}_{(n, m), Y, f}(\Lambda):=\sum_{\left(n^{\prime}, m^{\prime}\right) \in \mathcal{S}_{n, m}(\Omega)} f\left(\rho_{n^{\prime}, m^{\prime}} / \Lambda\right)
$$

for an even test function $f \in \mathcal{S}(\mathbb{R})$.

These eigenvalues have a structure similar to the spectrum of the Dirac operator on a torus, hence the computation of the action functional (4.24) can be approached via a Poisson summation formula.

Acknowledgement. The first author is supported by NSF grants DMS-1201512 and PHY1205440. The second author is supported by a Summer Undergraduate Research Fellowship at Caltech. We thank Kevin Iga for a careful reading of the manuscript and for very useful comments and suggestions.

\section{REFERENCES}

[1] A.M. Baranov, Yu.I. Manin, I.V. Frolov, A.S. Schwarz, A superanalog of the Selberg trace formula and multiloop contributions for fermionic strings, Commun. Math. Phys. Vol.111 (1987) N.3, 373-392.

[2] W. Beenakker, T. van den Broek, W. van Suijlekom, Supersymmetry and Noncommutative Geometry, Springer, 2015.

[3] G. Belyi, On Galois extensions of a maximal cyclotomic field, Math. USSR Izv. 14 (1980) N.2, 247-256. 
[4] J. Bolte, H.M. Stiepan, The Selberg trace formula for Dirac operators, J. Math. Phys. 47 (2006) N.11, $112104,16 \mathrm{pp}$.

[5] A.H. Chamseddine, A. Connes, The spectral action principle, Comm. Math. Phys. 186 (1997), no. 3, $731-750$

[6] C.H. Chang, D. Mayer, Thermodynamic formalism and Selberg's zeta function for modular groups, Regul. Chaotic Dyn., Vol.5 (2000) 281-312.

[7] D. Cimasoni, N. Reshetikhin, Dimers on surface graphs and spin structures, I, Comm. Math. Phys., Vol. 275 (2007) 187-208.

[8] P.B. Cohen, C. Itzykson, J. Wolfart, Fuchsian triangle groups and Grothendieck dessins. Variations on a theme of Belyi, Comm. Math. Phys. 163 (1994) N.3, 605-627.

[9] A. Connes, Geometry from the spectral point of view, Lett. Math. Phys. 34 (1995), no. 3, $203-238$.

[10] C.F. Doran, M.G. Faux, S.J. Gates, Jr., T. Hübsch, K.M. Iga, G.D. Landweber, R.L. Miller, Codes and supersymmetry in one dimension, Adv. Theor. Math. Phys., Vol.15 (2011) N.6, 1909-1970.

[11] C.F. Doran, M.G. Faux, S.J. Gates, Jr., T. Hübsch, K.M. Iga, G.D. Landweber, Relating doubly-even error-correcting codes, graphs, and irreducible representations of $N$-supersymmetry. in "Discrete and computational mathematics", pp.53-71, Nova Sci. Publ., 2008.

[12] C.F. Doran, M.G. Faux, S.J. Gates, Jr., T. Hübsch, K.M. Iga, G.D. Landweber, On graph-theoretic identifications of Adinkras, supersymmetry representations and superfields, Int. J. Mod. Phys. A 22 (2007) 869-930.

[13] C. Doran, K. Iga, G. Landweber, S. Méndez-Diez, Geometrization of N-extended 1-dimensional supersymmetry algebras, arXiv:1311.3736

[14] C.F. Doran, T. Gannon, H. Movasati, K.M. Shokri, Automorphic forms for triangle groups, Commun. Number Theory Phys. 7 (2013), no. 4, 689-737.

[15] M. Faux, S.J. Gates, Jr., Adinkras: a graphical technology for supersymmetric representation theory, Phys. Rev. D 71(3) (2005), 065002.

[16] J. Fisher, An approach to the Selberg trace formula via the Selberg zeta-function, Lecture Notes in Mathematics, Vol.1253, Springer, 1987.

[17] D. Fried, Symbolic dynamics for triangle groups, Invent. Math. 125 (1996) N. 3, 487-521.

[18] S.J. Gates Jr., T. Hübsch, On Dimensional Extension of Supersymmetry: From Worldlines to Worldsheets, Adv. in Th. Math. Phys., Vol.16 (2012) 1619-1667.

[19] E. Girondo, G. González-Diez, Introduction to compact Riemann surfaces and Dessins d'Enfants, Cambridge University Press, 2012.

[20] C. Grosche, Selberg supertrace formula for super Riemann surfaces, analytic properties of Selberg super zeta-functions and multiloop contributions for the fermionic string, Comm. Math. Phys. 133 (1990), no. 3, 433-485.

[21] C. Grosche, Path Integrals, hyperbolic spaces and Selberg trace formulae, World Scientific, 2013.

[22] R. Haag, M. Sohnius, J.Lopuszanski, All possible generators of supersymmetries of the S-matrix, Nuclear Physics B 88 (1975) 257-274.

[23] F. Herrlich, Introduction to origamis in Teichmüller space, in "Strasbourg master class on geometry", pp. 233-253, IRMA Lect. Math. Theor. Phys., 18, Eur. Math. Soc., Zürich, 2012.

[24] F. Herrlich, G. Schmithüsen, Dessins d'enfants and origami curves, Handbook of Teichmüller theory. Vol. II, 767-809, IRMA Lect. Math. Theor. Phys., 13, Eur. Math. Soc., 2009.

[25] T. Hübsch, Weaving worldsheet supermultiplets from the worldlines within, Adv.Theor. Math. Phys., Vol.17 (2013) N.5, 903-974.

[26] K. Iga, Y.X. Zhang, Structural theory of 2-d adinkras, arXiv:1508.00491.

[27] H. Kim, I. Saberi, Real homotopy theory and supersymmetric quantum mechanics, arXiv:1511.00978

[28] S.K. Lando, A.K. Zvonkin, Graphs on surfaces and their applications, With an appendix by Don B. Zagier. Encyclopaedia of Mathematical Sciences, 141. Low-Dimensional Topology, II. Springer-Verlag, Berlin, 2004.

[29] A. López Almorox, C. Tejero Prieto, Holomorphic spectrum of twisted Dirac operators on compact Riemann surfaces, J. Geom. Phys. 56 (2006), no. 10, 2069-2091.

[30] Yu.I. Manin, Topics in Noncommutative Geometry, Princeton University Press, 1991

[31] Yu.I. Manin, Gauge Field Theory and Complex Geometry, Springer, 1997.

[32] Yu.I. Manin, M. Marcolli, Continued fractions, modular symbols, and noncommutative geometry, Selecta Math. (N.S.) Vol.8 (2002) N.3, 475-521. 
[33] M. Marcolli, Limiting modular symbols and the Lyapunov spectrum, J. Number Theory, Vol.98 (2003) N.2, 348-376.

[34] M. Marcolli, Noncommutative Cosmology, book in preparation.

[35] M. Matone, Uniformization theory and 2D gravity. I. Liouville action and intersection numbers, Internat. J. Modern Phys. A 10 (1995) N.3, 289-335.

[36] M. Matone, Eigenfunctions of the Laplacian acting on degree zero bundles over special Riemann surfaces, Trans. Amer. Math. Soc. Vol.356 (2004) N.8, 2989-3004.

[37] D. Mayer, T. Mühlenbruch, F. Strömberg, The transfer operator for the Hecke triangle groups, Discrete Contin. Dyn. Syst. 32 (2012), no. 7, 2453-2484.

[38] M. Möller, Teichmüller curves, Galois actions and $\widehat{G T}$-relations, Mathematische Nachrichten 278 (2005) N.9, 1061-1077.

[39] M. Möller, A.D. Pohl, Period functions for Hecke triangle groups, and the Selberg zeta function as a Fredholm determinant, Ergodic Theory Dynam. Systems, Vol.33 (2013) N.1, 247-283.

[40] F. Nisbach, The Galois action on Origami curves and a special case of Origamis, PhD thesis, Karlsruher Institut für Technologie, 2011

[41] A. Pohl, Symbolic dynamics for the geodesic flow on two-dimensional hyperbolic good orbifolds, Discrete Contin. Dyn. Syst., Vol.34 (2014) N.5, 2173-2241.

[42] W. Roelcke, Das Eigenwertproblem der automorphen Formen in der hyperbolischen Ebene I, Math. Ann. 167 (1966) 292-337.

[43] G.B. Shabat, V.A. Voevodsky, Drawing curves over number fields, in The Grothendieck Festschrift, Vol. 3, Birkhäuser, 1990, pp. 199-227.

[44] L. Schneps, (Ed.) The Grothendieck theory of Dessins d'Enfants, Cambridge University Press, 1994.

[45] F. Strömberg, Computation of Selberg zeta functions on Hecke triangle groups, arXiv:0804.4837

[46] W. van Suijlekom, Noncommutative Geometry and Particle Physics, Springer 2014

[47] Y.X. Zhang, Adinkras for Mathematicians, Trans. Amer. Math. Soc., Vol.366 (2014) N.6, 3325-3355.

Division of Physics, Mathematics, and Astronomy, California institute of Technology, 1200 E California Blvd, Pasadena, CA 91125, USA

E-mail address: matilde@caltech.edu

E-mail address: nzolman@caltech.edu 